\title{
Analytical expressions for fringe fields in multipole magnets
}

\author{
B. D. Muratori, ${ }^{1,3}$ J. K. Jones, ${ }^{1,3}$ and A. Wolski ${ }^{2,3}$ \\ ${ }^{1}$ ASTeC, Sci-Tech Daresbury, Keckwick Lane, Daresbury, Warrington WA4 4AD, United Kingdom \\ ${ }^{2}$ Department of Physics, University of Liverpool, Liverpool, \\ L69 7ZE, United Kingdom \\ ${ }^{3}$ Cockcroft Institute, Sci-Tech Daresbury, Keckwick Lane, Daresbury, \\ Warrington WA4 4AD, United Kingdom \\ (Received 17 October 2014; published 5 June 2015)
}

\begin{abstract}
Fringe fields in multipole magnets can have a variety of effects on the linear and nonlinear dynamics of particles moving along an accelerator beam line. An accurate model of an accelerator must include realistic models of the magnet fringe fields. Fringe fields for dipoles are well understood and can be modeled at an early stage of accelerator design in such codes as MAD8, MADX, GPT or ELEGANT. Existing techniques for quadrupole and higher order multipoles rely either on the use of a numerical field map, or on a description of the field in the form of a series expansion about a chosen axis. Usually, it is not until the later stages of a design project that such descriptions (based on magnet modeling or measurement) become available. Furthermore, series expansions rely on the assumption that the beam travels more or less on axis throughout the beam line; but in some types of machines (for example, Fixed Field Alternating Gradients or FFAGs) this is not a good assumption. Furthermore, some tracking codes, such as GPT, use methods for including space charge effects that require fields to vary smoothly and continuously along a beam line: in such cases, realistic fringe field models are of significant importance. In this paper, a method for constructing analytical expressions for multipole fringe fields is presented. Such expressions allow fringe field effects to be included in beam dynamics simulations from the start of an accelerator design project, even before detailed magnet design work has been undertaken. The magnetostatic Maxwell equations are solved analytically and a solution that fits all orders of multipoles is derived. Quadrupole fringe fields are considered in detail as these are the ones that give the strongest effects. The analytic expressions for quadrupole fringe fields are compared with data obtained from numerical modeling codes in two cases: a magnet in the high luminosity upgrade of the Large Hadron Collider inner triplet, and a magnet in the nonscaling FFAG EMMA. In both examples, the analytical expressions provide a good approximation to the numerical field maps.
\end{abstract}

\section{INTRODUCTION}

Fringe fields represent regions that lie at the edges of a magnet where there is a transition from the nominal field to zero field, or to the field in an adjacent magnet. In multipole magnets, the nominal field has no longitudinal component. However, in the fringe field region where the fields vary with longitudinal position, Maxwell's equations require the presence of a nonzero longitudinal field component. For dipoles, the nominal field has only a single component. In dipole fringe fields, therefore, the field has only two components, and (assuming that the fields are independent of the horizontal transverse coordinate) analytical expressions for the field can be obtained by solving the twodimensional Laplace equation. For quadrupoles and higher-order multipoles, however, the fields in the fringe region have three components, and analytical expressions

Published by the American Physical Society under the terms of the Creative Commons Attribution 3.0 License. Further distribution of this work must maintain attribution to the author(s) and the published article's title, journal citation, and DOI. for these fields must be obtained by solving the threedimensional Laplace equation. Fringe fields can impact the motion of particles passing through magnets in a number of ways. For example, they can introduce nonlinearities in the equation of motion, or they can make a substantial contribution to the desired effects of the nominal field. The latter situation is the case in EMMA, for example, where the large aperture of the quadrupoles compared to their lengths means that the fringe fields dominate the focusing effects of these magnets. Nonlinearities from fringe fields can become important if the transverse size of the beam is large, or if the beam traverses a multipole at an angle and some distance from the magnetic axis: this is often the case in final focus quadrupoles in colliders. The implementation of fringe fields is also important in some tracking codes which include effects such as space charge, as is the case in GPT (General Particle Tracer) [1], for example. This requires that all fields be continuous so that there are smooth regions where the fields transit from their maximal value to zero and vice versa.

There are several models available for the study of fringe fields for multipoles, see for example [2-7] and references 
therein. However, existing models are usually limited to onaxis and midplane approximations, meaning that the field in the full space of the multipole typically has to be computed with elliptic integrals. Therefore, for simple but accurate particle tracking, a significant amount of effort and computing power goes into just the calculation of the fields that particles see. The results obtained in this paper make possible an alternative method, based on analytical expressions for the fields as functions of position, which provide exact solutions to the static Maxwell equations in three dimensions. This allows for arbitrarily smooth fields to be constructed and used in tracking codes as well as the possible creation of transfer maps. The fringe fields considered here have associated scalar and vector potentials. From the scalar potential it is possible to inspect the shape of the pole face for an iron-dominated magnet generating the given field. Using the vector potential it is possible to perform symplectic integration of the equations of motion for a particle in the field, leading to the construction of transfer maps for the fringe region. Further, it may be possible to improve the efficiency of the magnet design process by making some initial assumptions based on the formulas presented in this paper. However, a full three-dimensional numerical field map will ultimately be needed (obtained from a numerical magnet modeling code) in order to achieve the accuracy that is needed for validating the design of many accelerators.

This paper develops the mathematical framework that was initially presented in [8], gives further details and results, and presents two examples. In Sec. II, fringe fields for dipoles are briefly reviewed. The formalism used for dipoles is extended to fully three-dimensional fields in Sec. III. A complete solution to the static Maxwell equations, in a form suitable for application to multipole fringe fields, is derived and presented. Expressions for fringe fields in multipoles of arbitrary order are then given in Sec. IV. A particular case of a quadrupole fringe field, with a simple falloff in the form of an Enge function [9], is then presented in Sec. V. All the salient properties are described in order to demonstrate that the field behaves in the way expected of a quadrupole, first inside the magnet, then in the fringe field region, and finally at a large distance from the magnet (so that the field effectively falls to zero). In Sec. VI, the scalar and vector potentials for fringe fields in the case of a multipole of arbitrary order are discussed. In Sec. VII, two quadrupole examples are presented. The first is the high luminosity upgrade of the Large Hadron Collider (HL-LHC) inner triplet, where the beam size and trajectory in the quadrupoles providing strong focusing close to the interaction point make fringe field effects significant. The second example is based on the quadrupole magnets in the nonscaling Fixed Field Alternating Gradient (FFAG), EMMA. The large aperture of these magnets compared to their length means that the fringe fields make a dominant contribution to the focusing effects. Conclusions are given in Sec. VIII.

\section{FRINGE FIELDS FOR DIPOLES}

The goal is to derive expressions for multipole fringe fields that satisfy Maxwell's equations. To ensure the validity of the solution and the corresponding assumptions, it is important to write all equations explicitly. For static fields in the absence of any electric current, the equations for the magnetic field $\vec{B}$ are

$$
\nabla \times \vec{B}=\nabla \cdot \vec{B}=0 .
$$

For dipole magnets, it is sufficient to consider a twodimensional version of the equations. Taking $B_{x}=0$, we are left with

$$
\partial_{y} B_{y}+\partial_{z} B_{z}=\partial_{y} B_{z}-\partial_{z} B_{y}=0
$$

together with

$$
\partial_{x} B_{z}=\partial_{x} B_{y}=0,
$$

which excludes all dependence on $x$. Maxwell's equations (1) imply

$$
\Delta_{y, z} B_{y}=\Delta_{y, z} B_{z}=0,
$$

where $\Delta_{y, z}=\partial_{y}^{2}+\partial_{z}^{2}$. Both equations (for $B_{y}$ and $B_{z}$ ) can easily be solved:

$$
\begin{aligned}
B_{y} & =f_{y}(z+i y)+g_{y}(z-i y), \\
B_{z} & =f_{z}(z+i y)+g_{z}(z-i y),
\end{aligned}
$$

for arbitrary functions $f_{y}, g_{y}, f_{z}$ and $g_{z}$. Requiring that equations (1) be solved as well, we end up with

$$
\begin{aligned}
& B_{y}=f(z+i y)+g(z-i y), \\
& B_{z}=-i f(z+i y)+i g(z-i y),
\end{aligned}
$$

for arbitrary functions $f$ and $g$. If we further restrict ourselves to real magnetic fields, we obtain

$$
\begin{gathered}
B_{y}=f(z+i y)+\bar{f}(z-i y), \\
B_{z}=-i f(z+i y)+i \bar{f}(z-i y),
\end{gathered}
$$

where the function $\bar{f}$ is the complex conjugate of the function $f . B_{y}$ and $B_{z}$ are given by twice the real and imaginary parts of the function $f(z+i y)$, respectively.

As an illustration of these results, consider the following form for dipole fringe fields (that is used, for example, in the code GPT [10]): 


$$
\begin{gathered}
B_{y}=\frac{B_{0}}{2 \pi}\left[\pi-\arctan \left(\frac{z}{\alpha+y}\right)-\arctan \left(\frac{z}{\alpha-y}\right)\right], \\
B_{z}=\frac{B_{0}}{4 \pi}\left\{\ln \left[z^{2}+(\alpha+y)^{2}\right]-\ln \left[z^{2}+(\alpha-y)^{2}\right]\right\},
\end{gathered}
$$

where $B_{0}$ is the nominal strength of the dipole field, and $\alpha$ is a parameter (related to the aperture of the magnet) affecting the precise shape of the fringe field. Using the result

$$
[z+i(\alpha \pm y)][z-i(\alpha \pm y)]=z^{2}+(\alpha \pm y)^{2},
$$

the expression for $B_{z}$ in (6) may be rewritten as

$$
\begin{aligned}
B_{z}= & \frac{B_{0}}{4 \pi}\{\ln [z+i(\alpha+y)]+\ln [z-i(\alpha+y)] \\
& -\ln [z+i(\alpha-y)]-\ln [z-i(\alpha-y)]\},
\end{aligned}
$$

whence $f(z+i y)$ has the form

$$
f(z+i y)=\frac{i B_{0}}{4 \pi}[\ln (z+i y+i \alpha)-\ln (z+i y-i \alpha)] .
$$

Then, using Eq. (3) we find $B_{y}$ to be given by

$$
\begin{aligned}
B_{y}= & \frac{i B_{0}}{4 \pi}\{\ln [z+i(\alpha+y)]-\ln [z-i(\alpha+y)] \\
& +\ln [z+i(\alpha-y)]-\ln [z-i(\alpha-y)]\},
\end{aligned}
$$

which may be converted into the same form as $B_{y}$ given in (5).

As a second illustration, consider a magnetic field in which the $B_{y}$ component varies along the $z$ axis as an Enge function [9]:

$$
\left.B_{y}\right|_{y=0}=\frac{1}{1+e^{E(z)}},
$$

with $E(z)$ given by

$$
E(z)=\sum_{n=0}^{N-1} a_{n}\left(\frac{z}{D}\right)^{n}
$$

for some set of $N$ coefficients $a_{n}$. D is the aperture of the dipole, and for simplicity we consider a magnet with unit field strength (i.e., $B_{y}=1$ in the body of the magnet). In general, the number of terms in the summation (8) can be chosen to customize the shape of the falloff of the field, with the values of the coefficients determined by modeling or measurement. For example, a six-parameter Enge function is used in [11]. The main advantage of the Enge function is that it is analytic and can be made to tend to asymptotic values arbitrarily fast. The main disadvantage is that, if several coefficients $a_{n}$ with $N>1$ are included, features of the fringe field cannot readily be associated with particular coefficients. For example, the "hardness" of the fringe field (the distance taken for the field to fall below a given value) depends on the combined effect of all the coefficients. Other functions that decay sufficiently rapidly may be used instead of the Enge function $[12,13]$. For simplicity, we consider in this paper only Enge functions where $a_{1} \neq 0$ and all other coefficients are set to zero. Because we only have one nonzero coefficient, we can normalize it to unit magnet aperture (effectively replacing $a_{1}$ with $a_{1} / D$ ) in (8) without loss of generality.

Given a dipole fringe field (7) with Enge-type falloff on the $z$ axis, the results (3) and (4) imply that the dependence of $B_{y}$ on coordinates $y$ and $z$ can be written

$$
B_{y}=\frac{1}{2\left(1+e^{E(z+i y)}\right)}+\frac{1}{2\left(1+e^{E(z-i y)}\right)} .
$$

Furthermore, $B_{z}$ has the form

$$
B_{z}=\frac{-i}{2\left(1+e^{E(z+i y)}\right)}+\frac{i}{2\left(1+e^{E(z-i y)}\right)},
$$

where the complex function $E(z+i y)$ is obtained by replacing $z$ with $z+i y$ in (8). If we consider the simple case $E(z+i y)=z+i y$ (i.e., a single-parameter Enge function with $a_{1}=1$ ) then Eqs. (9) and (10) simplify to

$$
\begin{aligned}
& B_{y}=\frac{1+e^{z} \cos y}{1+2 e^{z} \cos y+e^{2 z}}, \\
& B_{z}=\frac{-e^{z} \sin y}{1+2 e^{z} \cos y+e^{2 z}} .
\end{aligned}
$$

This may be extended to include as many parameters of the Enge function as desired. So, if $E(z+i y)=$ $a_{1}(z+i y)+a_{2}(z+i y)^{2}$, with $a_{1}$ and $a_{2}$ arbitrary constants, we have

$$
\begin{aligned}
& B_{y}=\frac{1+e^{\theta} \cos \psi}{1+2 e^{\theta} \cos \psi+e^{2 \theta}}, \\
& B_{z}=\frac{-e^{\theta} \sin \psi}{1+2 e^{\theta} \cos \psi+e^{2 \theta}},
\end{aligned}
$$

where $\theta=a_{1} z+a_{2}\left(z^{2}-y^{2}\right)$ and $\psi=y\left(a_{1}+2 a_{2} z\right)$.

We can plot the fringe fields (9) and (10) in the simplest case $E(z+i y)=z+i y$, as shown in Fig. 1. Each field component has singularities, of which two are visible in the plots. From the denominator of (9) and (10), it can be seen that the singularities appear when (reintroducing the arbitrary aperture diameter $D$ )

$$
1+2 e^{z / D} \cos (y / D)+e^{2 z / D}=0 .
$$

Hence, singularities occur at vertical positions: 

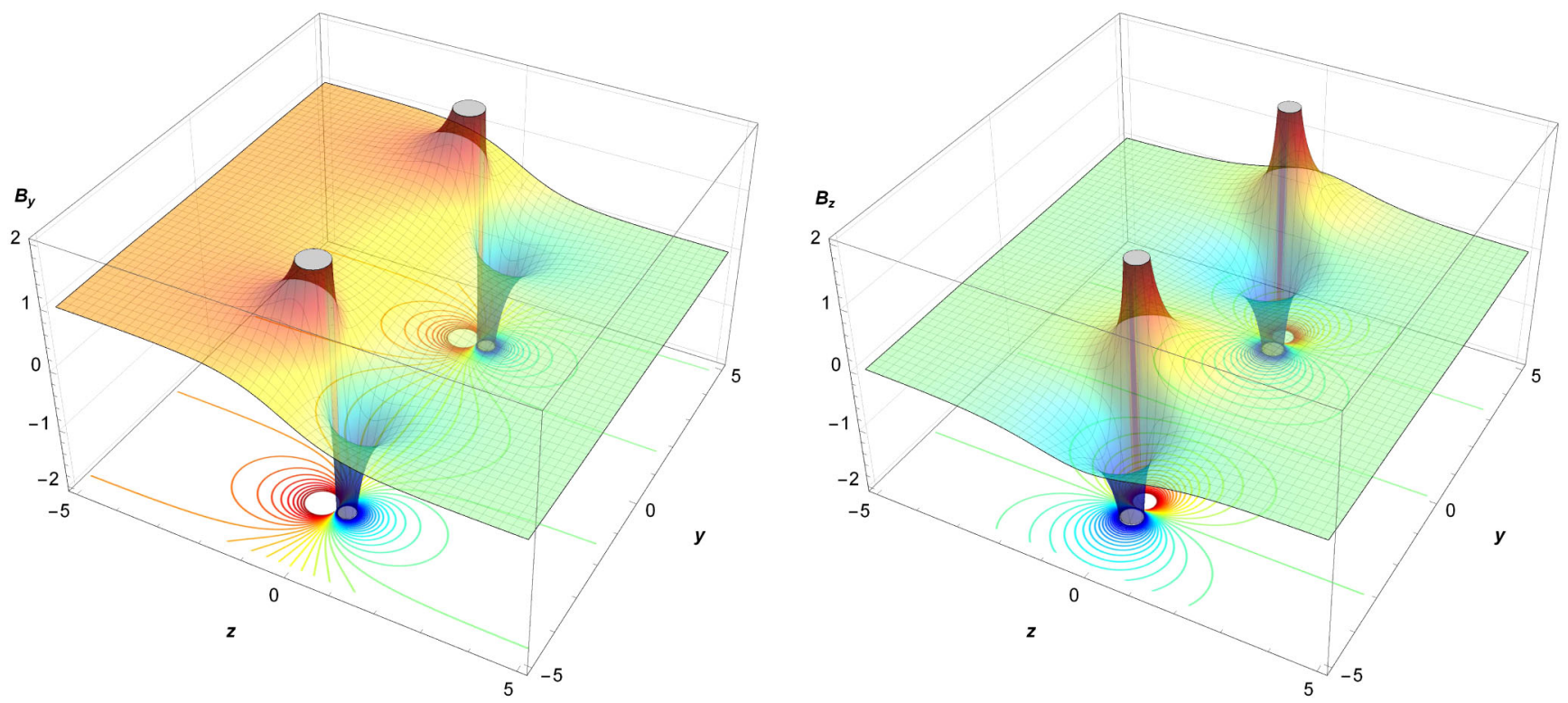

FIG. 1. Field components $B_{y}$ and $B_{z}$ in the fringe field region of a dipole magnet. The falloff of the field in this case in the plane $y=0$ is given by a single-parameter Enge function.

$$
y= \pm \ell \pi D
$$

where $\ell$ is an odd integer. The presence of singularities does not cause any problems for modeling the field in practice, as it is always possible to arrange that the singularities are located outside the region of interest. It is not possible to avoid the appearance of singularities since any solution to Laplace's equation that is bounded at infinity either has singularities or is constant. In practical terms, the singularities can be associated with the currents acting as sources of the magnetic field. The singularities have a tanlike behavior: at the exact point where $z=0$, the value of the field is precisely half of the maximum and everything is smooth. This can be verified by setting $z=0$ in the expressions for the dipole fringe fields above and using l'Hopital's rule.

Locally, it is possible to find potentials for the dipole fringe fields. For magnetostatic fields in the absence of currents, the field can be derived from a scalar potential $\varphi$, by

$$
\vec{B}=\nabla \varphi \text {. }
$$

The scalar potential exists because $\nabla \times \vec{B}=0$. For the simple case discussed above, the scalar potential is given by

$\varphi=y+\frac{i}{2} \ln \left(1+e^{z+i y}\right)-\frac{i}{2} \ln \left(1+e^{z-i y}\right)+$ constant,

with the only gauge freedom being given by the constant. From the scalar potential, it is possible to obtain a description of the pole-face geometry, since this is given by surfaces where $\varphi$ is constant. This is shown in Fig. 2 where two profiles of the pole face can be seen. It should be remembered that there is a scale invariance in the expressions depending on the dimensions of the dipole. Figure 2 also shows that the pole-face profiles encompass the singularities: this is consistent with the assertion made earlier that the singularities are associated with currents acting as sources of the field.

The usual vector potential $\vec{A}$ is related to the field by

$$
\vec{B}=\nabla \times \vec{A}
$$

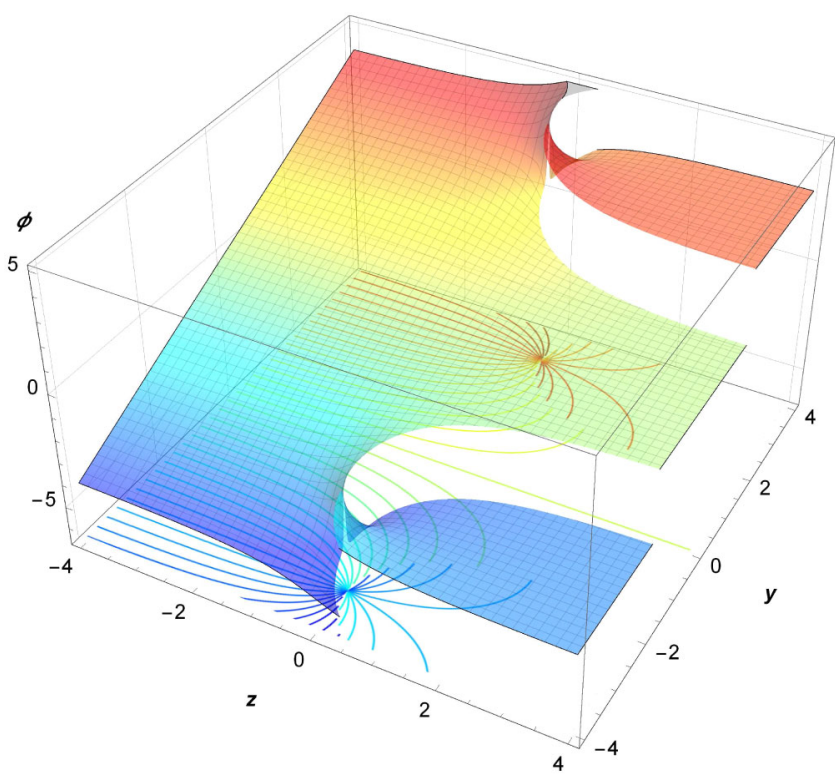

FIG. 2. Scalar potential $\varphi$ in the fringe field region of a dipole magnet. The field has a falloff in the plane $y=0$ described by a single-parameter Enge function, the contours of constant $\varphi$ showing possible pole-face profiles. 
The existence of the vector potential is ensured by the Maxwell equation $\nabla \cdot \vec{B}=0$. The vector potential has extensive gauge freedom, but in one of its simplest forms the vector potential in a dipole fringe field can be given as

$$
A_{x}=z-\frac{1}{2} \ln \left(1+e^{z+i y}\right)-\frac{1}{2} \ln \left(1+e^{z-i y}\right),
$$

with the other components of $\vec{A}$ set to zero.

\section{ELEMENTARY SOLUTION IN A FRINGE FIELD}

General expressions for the fringe fields in a dipole followed from writing Maxwell's equations in the form (1). In a dipole, we only needed to consider two field components $\left(B_{y}\right.$ and $\left.B_{z}\right)$ as functions of two coordinates ( $y$ and $z$ ). For higher order multipoles, it is necessary to consider the dependence of all three field components on all three coordinates. One approach might be to look for solutions to the three-dimensional Laplacian:

$$
\Delta_{x, y, z} \varphi \equiv \partial_{x}^{2} \varphi+\partial_{y}^{2} \varphi+\partial_{z}^{2} \varphi=0,
$$

and obtain the field components from $\vec{B}=\nabla \varphi$. A formal solution (due to Whittaker [14-16]; also given, for example, in [17]) is known, and can be expressed as

$$
\varphi(x, y, z)=f(z+i x \cos \vartheta+i y \sin \vartheta),
$$

where $f$ is an arbitrary analytic function, and $\vartheta$ is an arbitrary parameter (that can take complex as well as real values). Of course, it is possible to superpose solutions of the form (17) with different values of $\vartheta$ to obtain solutions to Laplace's equation in particular cases. For example, the potential

$\varphi(x, y, z)=\frac{1}{2 \pi} \int_{0}^{2 \pi} \frac{\mathrm{d} \vartheta}{z+i x \cos \vartheta+i y \sin \vartheta}=\frac{1}{\sqrt{x^{2}+y^{2}+z^{2}}}$

represents the electrostatic potential around a point charge. It is also possible to use (17) to construct potentials from which fields may be derived with the properties expected of fringe fields in multipole magnets. In this paper, we give a derivation of suitable expressions for the fields in such cases; however, rather than starting from (17) we start from Maxwell's equations for magnetostatic fields in free space. This leads to somewhat different expressions for the fields than would naturally be derived from (17), although it is straightforward to show that the expressions resulting from the two starting points are directly related: this is discussed further in Sec. VI A. It is possible that different forms of the expressions for multipole fringe fields will be convenient for different cases. The examples that we consider in
Sec. VII are based on implementation of the expressions derived from solution of Maxwell's equations.

Our goal is to find solutions to Maxwell's equations that describe the field in the fringe field region of a multipole magnet. The basis of our approach is to define new variables in terms of which Maxwell's equations can be written for a general three-dimensional magnetostatic field in a form very similar to (1). This raises the possibility of finding expressions for fringe fields in higher order multipoles by applying similar techniques to those described in Sec. II for dipoles. To begin, we define new variables:

$$
\begin{gathered}
u=\frac{1}{\sqrt{2}}(x+i y), \\
v=\frac{1}{\sqrt{2}}(x-i y), \\
\zeta=\sqrt{2} z .
\end{gathered}
$$

We express the magnetic field in terms of components:

$$
\begin{gathered}
B_{u}=\frac{1}{\sqrt{2}}\left(B_{x}+i B_{y}\right), \\
B_{v}=\frac{1}{\sqrt{2}}\left(B_{x}-i B_{y}\right), \\
B_{\zeta}=\frac{1}{\sqrt{2}} B_{z} .
\end{gathered}
$$

In terms of the new variables, the magnetostatic Maxwell's equations can be written:

$$
\begin{aligned}
& \partial_{u} B_{u}+\partial_{\zeta} B_{\zeta}=0, \\
& \partial_{v} B_{v}+\partial_{\zeta} B_{\zeta}=0, \\
& \partial_{\zeta} B_{u}-\partial_{v} B_{\zeta}=0, \\
& \partial_{\zeta} B_{v}-\partial_{u} B_{\zeta}=0 .
\end{aligned}
$$

From (24) and (25), one can see immediately that, in the absence of any fringe fields, the general solution of Maxwell's equations for any magnet, acting transversely only and without fringe $\left(B_{\zeta}=0\right)$ is given by

$$
\begin{aligned}
& B_{u}=B_{u}(v), \\
& B_{v}=B_{v}(u),
\end{aligned}
$$

for any function $B_{u}(v)$ (that is a function of $v$ only) and $B_{v}(u)$ (that is a function of $u$ only). The case of a multipole of order $n$ ( $n=0$ for a dipole, $n=1$ for a quadrupole, $n=2$ for a sextupole, and so on) is given by 


$$
\begin{gathered}
B_{u}=i v^{n}, \\
B_{v}=-i u^{n}, \\
B_{\zeta}=0 .
\end{gathered}
$$

Using (18)-(23), we find that the transverse components of the field in this case in Cartesian coordinates are

$$
\begin{aligned}
& B_{x}=2^{\frac{1-n}{2}} \sin (n \theta), \\
& B_{y}=2^{\frac{1-n}{2}} \cos (n \theta),
\end{aligned}
$$

where $\theta$ is the azimuthal angle with respect to the $x$ axis. In cylindrical polar coordinates, the radial field component is

$$
B_{r}=2^{\frac{1-n}{2}} \sin ((n+1) \theta),
$$

from which we see that the field given by (28)-(30) has the behavior of a multipole of order $n$.

To describe a multipole fringe field, we need to include a dependence of the fields on the longitudinal coordinate $\zeta$. One way to proceed is to write down expressions for the fields (as functions of $u, v$ and $\zeta$ ) with a high degree of generality, but with a structure based on a generalization of the fringe fields for the dipole case. Substituting the assumed expressions into Maxwell's equations leads to explicit constraints on the form that the expressions for the fields can take: however, if the initial assumption is sufficiently general, the constraints allow the description of a field that represents a multipole (of any given order) in the body of a magnet, but that decays in any desired fashion along the axis of the magnet with increasing distance from the magnet (i.e., with increasing $\zeta$ ). Details of this process are given in the Appendix. The result is a solution to Maxwell's equations of the following form:

$$
\begin{gathered}
B_{u}=i b f(\zeta+i h)-i b g(\zeta-i h), \\
B_{v}=\frac{i}{b} f(\zeta+i h)-\frac{i}{b} g(\zeta-i h), \\
B_{\zeta}=f(\zeta+i h)+g(\zeta-i h),
\end{gathered}
$$

where $f$ and $g$ are arbitrary functions, $b$ is an arbitrary parameter (which can take real or complex values), and $h$ is defined by

$$
h=\frac{u}{b}+b v .
$$

For the field (31)-(33) to describe a physical fringe field for a multipole magnet, additional constraints must be imposed on the functions $f$ and $g$. Furthermore, it will be necessary to superpose solutions with different values of the parameter $b$. We therefore refer to (31)-(33) as the "elementary" solution (in the sense that it provides the basic elements for constructing physical multipole fringe fields). In the following sections, we discuss how physical multipole fringe fields can be constructed based on the elementary solution, and provide some examples.

\section{MULTIPOLE FRINGE FIELDS WITH ARBITRARY FALLOFF}

From inspection of the elementary solution (31)-(33) we see that $B_{u} \propto B_{v}$ : no physical magnetic fields can be represented this way. However, because of the linearity of Maxwell's equations, any linear combination of elementary solutions gives a solution to Maxwell's equations. Physical solutions corresponding to multipole fields can be constructed by taking appropriate combinations of elementary solutions. Further (physical) constraints are that the field decays to zero as $\zeta \rightarrow \infty$ and that the field matches the nominal multipole field inside the magnet. In this section, we describe in detail the procedure for constructing an expression for the fringe field in a quadrupole, and then generalize our results to multipoles of any order.

To obtain the correct behavior of the field as a function of $\zeta$, the functions $f(\zeta+i h)$ and $g(\zeta-i h)$ are each written as a product of two factors. The first factor, $(\zeta+i h)^{n}$ and $(\zeta-i h)^{n}$ for $f$ and $g$ respectively, represents the (nominal) multipole for some constant $n$. The second factor, $F(\zeta+i h)$ and $G(\zeta-i h)$ for $f$ and $g$ respectively, is a multiplicative function that is chosen to give the desired decay as $\zeta$ becomes large $(\zeta \rightarrow \infty)$, and to give the correct field in the body of the magnet $(\zeta \rightarrow-\infty)$. Thus, we write

$$
\begin{gathered}
f(\zeta+i h)=(\zeta+i h)^{n} F(\zeta+i h), \\
g(\zeta-i h)=(\zeta-i h)^{n} G(\zeta-i h) .
\end{gathered}
$$

Following the form of (31)-(33), let $B_{u}, B_{v}$ and $B_{\zeta}$ be given by linear combinations of the elementary solution:

$$
\begin{aligned}
B_{u} & =\sum_{j=1}^{m} c_{j}\left[i b_{j}\left(\zeta+i h_{j}\right)^{n} F_{j}\left(\zeta+i h_{j}\right)-i b_{j}(\zeta-i h)^{n} G_{j}\left(\zeta-i h_{j}\right)\right], \\
B_{v} & =\sum_{j=1}^{m} c_{j}\left[\frac{i}{b_{j}}\left(\zeta+i h_{j}\right)^{n} F_{j}\left(\zeta+i h_{j}\right)-\frac{i}{b_{j}}\left(\zeta-i h_{j}\right)^{n} G_{j}\left(\zeta-i h_{j}\right)\right],
\end{aligned}
$$


$B_{\zeta}=\sum_{j=1}^{m} c_{j}\left[\left(\zeta+i h_{j}\right)^{n} F_{j}\left(\zeta+i h_{j}\right)+\left(\zeta-i h_{j}\right)^{n} G_{j}\left(\zeta-i h_{j}\right)\right]$,

where

$$
h_{j}=\frac{u}{b_{j}}+b_{j} v,
$$

and $m$ is a constant determined by the number of copies of the elementary solution needed to construct a physical multipole of the desired order. The constant $n$ determines the order of the multipole. Within the body of the magnet (i.e., in the limit $\zeta \rightarrow-\infty), B_{u}=i v^{n}, B_{v}=-i u^{n}$, and
$B_{\zeta}=0$ : these conditions impose a number of constraints on the constants $b_{j}$ and $c_{j}$, which are discussed in the remainder of this section. We first consider in turn quadrupole, sextupole and octupole magnets. The results in these cases establish a pattern that is readily generalized to multipole magnets of arbitrary order.

\section{A. Quadrupole magnets}

A quadrupole field is obtained by putting $n=1$ in Eqs. (35) and (36). Within the body of the magnet (far from any fringe field) $F_{j}\left(\zeta+i h_{j}\right)=G_{j}\left(\zeta-i h_{j}\right)=1$ for any $j$. The required behavior of the fields in the body of the magnet can be obtained by taking $m=2$ (larger values of $m$ can be used, but are not required). Then, putting $B_{u}=i v$, $B_{v}=-i u$ and $B_{\zeta}=0$ gives

$$
\begin{aligned}
i c_{1} b_{1}\left(\zeta+i \frac{u}{b_{1}}+i b_{1} v\right)-i c_{1} b_{1}\left(\zeta-i \frac{u}{b_{1}}-i b_{1} v\right)+i c_{2} b_{2}\left(\zeta+i \frac{u}{b_{2}}+i b_{2} v\right)-i c_{2} b_{2}\left(\zeta-i \frac{u}{b_{2}}-i b_{2} v\right) & =i v, \\
i \frac{c_{1}}{b_{1}}\left(\zeta+i \frac{u}{b_{1}}+i b_{1} v\right)-i \frac{c_{1}}{b_{1}}\left(\zeta-i \frac{u}{b_{1}}-i b_{1} v\right)+i \frac{c_{2}}{b_{2}}\left(\zeta+i \frac{u}{b_{2}}+i b_{2} v\right)-i \frac{c_{2}}{b_{2}}\left(\zeta-i \frac{u}{b_{2}}-i b_{2} v\right) & =-i u, \\
c_{1}\left(\zeta+i \frac{u}{b_{1}}+i b_{1} v\right)+ & c_{1}\left(\zeta-i \frac{u}{b_{1}}-i b_{1} v\right)+c_{2}\left(\zeta+i \frac{u}{b_{2}}+i b_{2} v\right)+c_{2}\left(\zeta-i \frac{u}{b_{2}}-i b_{2} v\right)
\end{aligned}
$$

Thus, we have three equations to satisfy:

$$
\begin{aligned}
2 c_{1} b_{1}^{2}+2 c_{2} b_{2}^{2} & =-i, \\
-2 \frac{c_{1}}{b_{1}^{2}}-2 \frac{c_{2}}{b_{2}^{2}} & =-i, \\
c_{1}+c_{2} & =0 .
\end{aligned}
$$

Therefore, inside the magnet, the coefficients $b_{j}$ and $c_{j}$ must satisfy the following constraints:

$$
\begin{gathered}
c_{1}=-\frac{i}{2\left(b_{1}^{2}-b_{2}^{2}\right)}, \\
c_{2}=-\frac{i}{2\left(b_{2}^{2}-b_{1}^{2}\right)}, \\
b_{1}= \pm \frac{1}{b_{2}} .
\end{gathered}
$$

We are left with the freedom of choosing one constant, which we take to be $b_{2}$. We shall consider the significance of this constant in more detail later, but for now we note that to avoid $b_{1}$ becoming singular, $b_{2}$ must not be equal to 0 ; and to avoid $c_{1}$ and $c_{2}$ becoming singular, $b_{2}$ must not be equal to \pm 1 or $\pm i$. Also, the field is unchanged if we replace $b_{2}$ by $1 / b_{2}$.
The results for fields in quadrupole magnets can be extended to higher order multipoles in a straightforward way. However, the higher the order, the more copies of the elementary solution are needed to construct fields with the required properties. In the following subsections, we shall consider explicitly the cases of sextupole and octupole magnets, to establish a pattern from which the results for a general multipole magnet can be written down.

\section{B. Sextupole magnets}

A sextupole field is obtained by putting $n=2$ in Eqs. (35) and (36). To obtain the constraints on the various coefficients $c_{j}$ and $b_{j}$, we can proceed in close analogy with the case of the quadrupole magnet. In particular, within the body of the magnet (far from any fringe field) $F_{j}\left(\zeta+i h_{j}\right)=1$ and $G_{j}\left(\zeta-i h_{j}\right)=-1$ for any $j$, and the field is given by

$$
\begin{aligned}
& B_{u}=i v^{2}, \\
& B_{v}=-i u^{2}, \\
& B_{\zeta}=0 .
\end{aligned}
$$

The corresponding constraints are 


$$
\begin{array}{r}
i c_{1} b_{1}\left(\zeta+i \frac{u}{b_{1}}+i b_{1} v\right)^{2}+i c_{1} b_{1}\left(\zeta-i \frac{u}{b_{1}}-i b_{1} v\right)^{2}+i c_{2} b_{2}\left(\zeta+i \frac{u}{b_{2}}+i b_{2} v\right)^{2}+i c_{2} b_{2}\left(\zeta-i \frac{u}{b_{2}}-i b_{2} v\right)^{2} \\
+i c_{3} b_{3}\left(\zeta+i \frac{u}{b_{3}}+i b_{3} v\right)^{2}+i c_{3} b_{3}\left(\zeta-i \frac{u}{b_{3}}-i b_{3} v\right)^{2}=i v^{2}, \\
i \frac{c_{1}}{b_{1}}\left(\zeta+i \frac{u}{b_{1}}+i b_{1} v\right)^{2}+i \frac{c_{1}}{b_{1}}\left(\zeta-i \frac{u}{b_{1}}-i b_{1} v\right)^{2}+i \frac{c_{2}}{b_{2}}\left(\zeta+i \frac{u}{b_{2}}+i b_{2} v\right)^{2}+i \frac{c_{2}}{b_{2}}\left(\zeta-i \frac{u}{b_{2}}-i b_{2} v\right)^{2} \\
+i \frac{c_{3}}{b_{3}}\left(\zeta+i \frac{u}{b_{3}}+i b_{3} v\right)^{2}+i \frac{c_{3}}{b_{3}}\left(\zeta-i \frac{u}{b_{3}}-i b_{3} v\right)^{2}=-i u^{2}, \\
c_{1}\left(\zeta+i \frac{u}{b_{1}}+i b_{1} v\right)^{2}-c_{1}\left(\zeta-i \frac{u}{b_{1}}-i b_{1} v\right)^{2}+c_{2}\left(\zeta+i \frac{u}{b_{2}}+i b_{2} v\right)^{2}-c_{2}\left(\zeta-i \frac{u}{b_{2}}-i b_{2} v\right)^{2} \\
+c_{3}\left(\zeta+i \frac{u}{b_{3}}+i b_{3} v\right)^{2}-c_{3}\left(\zeta-i \frac{u}{b_{3}}-i b_{3} v\right)^{2}=0 .
\end{array}
$$

There are four equations to satisfy, namely,

$$
\begin{aligned}
2 c_{1} b_{1}^{3}+2 c_{2} b_{2}^{3}+2 c_{3} b_{3}^{3} & =-1, \\
-2 \frac{c_{1}}{b_{1}^{3}}-2 \frac{c_{2}}{b_{2}^{3}}-2 \frac{c_{3}}{b_{3}^{3}} & =-1, \\
c_{1} b_{1}+c_{2} b_{2}+c_{3} b_{3} & =0 \\
\frac{c_{1}}{b_{1}}+\frac{c_{2}}{b_{2}}+\frac{c_{3}}{b_{3}} & =0 .
\end{aligned}
$$

Finally, the constraints on the coefficients $b_{j}$ and $c_{j}$ may be written

$$
\begin{aligned}
& c_{1}=-\frac{b_{1}}{2\left(b_{1}^{2}-b_{2}^{2}\right)\left(b_{1}^{2}-b_{3}^{2}\right)}, \\
& c_{2}=-\frac{b_{2}}{2\left(b_{2}^{2}-b_{1}^{2}\right)\left(b_{2}^{2}-b_{3}^{2}\right)}, \\
& c_{3}=-\frac{b_{3}}{2\left(b_{3}^{2}-b_{1}^{2}\right)\left(b_{3}^{2}-b_{2}^{2}\right)}, \\
& b_{1}= \pm \frac{i}{b_{2} b_{3}} .
\end{aligned}
$$

For a sextupole, there are two free parameters, which we can take to be $b_{2}$ and $b_{3}$.

\section{Octupole magnets}

An octupole field is obtained by putting $n=3$ in Eqs. (35) and (36). Within the body of the magnet $F_{j}\left(\zeta+i h_{j}\right)=G_{j}\left(\zeta-i h_{j}\right)=1$ for any $j$, and the field is given by

$$
\begin{aligned}
& B_{u}=i v^{3}, \\
& B_{v}=-i u^{3}, \\
& B_{\zeta}=0 .
\end{aligned}
$$

Following the same procedure as for the quadrupole and the sextupole, the constraints on the coefficients $c_{j}$ and $b_{j}$ are

$$
\begin{aligned}
& i c_{1} b_{1}\left(\zeta+i \frac{u}{b_{1}}+i b_{1} v\right)^{3}-i c_{1} b_{1}\left(\zeta-i \frac{u}{b_{1}}-i b_{1} v\right)^{3}+i c_{2} b_{2}\left(\zeta+i \frac{u}{b_{2}}+i b_{2} v\right)^{3}-i c_{2} b_{2}\left(\zeta-i \frac{u}{b_{2}}-i b_{2} v\right)^{3} \\
& +i c_{3} b_{3}\left(\zeta+i \frac{u}{b_{3}}+i b_{3} v\right)^{3}-i c_{3} b_{3}\left(\zeta-i \frac{u}{b_{3}}-i b_{3} v\right)^{3}+i c_{4} b_{4}\left(\zeta+i \frac{u}{b_{4}}+i b_{4} v\right)^{3}-i c_{4} b_{4}\left(\zeta-i \frac{u}{b_{4}}-i b_{4} v\right)^{3}=i v^{3} \text {, } \\
& i \frac{c_{1}}{b_{1}}\left(\zeta+i \frac{u}{b_{1}}+i b_{1} v\right)^{3}-i \frac{c_{1}}{b_{1}}\left(\zeta-i \frac{u}{b_{1}}-i b_{1} v\right)^{3}+i \frac{c_{2}}{b_{2}}\left(\zeta+i \frac{u}{b_{2}}+i b_{2} v\right)^{3}-i \frac{c_{2}}{b_{2}}\left(\zeta-i \frac{u}{b_{2}}-i b_{2} v\right)^{3} \\
& +i \frac{c_{3}}{b_{3}}\left(\zeta+i \frac{u}{b_{3}}+i b_{3} v\right)^{3}-i \frac{c_{3}}{b_{3}}\left(\zeta-i \frac{u}{b_{3}}-i b_{3} v\right)^{3}+i \frac{c_{4}}{b_{4}}\left(\zeta+i \frac{u}{b_{4}}+i b_{4} v\right)^{3}-i \frac{c_{4}}{b_{4}}\left(\zeta-i \frac{u}{b_{4}}-i b_{4} v\right)^{3}=-i u^{3} \text {, } \\
& c_{1}\left(\zeta+i \frac{u}{b_{1}}+i b_{1} v\right)^{3}+c_{1}\left(\zeta-i \frac{u}{b_{1}}-i b_{1} v\right)^{3}+c_{2}\left(\zeta+i \frac{u}{b_{2}}+i b_{2} v\right)^{3}+c_{2}\left(\zeta-i \frac{u}{b_{2}}-i b_{2} v\right)^{3} \\
& +c_{3}\left(\zeta+i \frac{u}{b_{3}}+i b_{3} v\right)^{3}+c_{3}\left(\zeta-i \frac{u}{b_{3}}-i b_{3} v\right)^{3}+c_{4}\left(\zeta+i \frac{u}{b_{4}}+i b_{4} v\right)^{3}+c_{4}\left(\zeta-i \frac{u}{b_{4}}-i b_{4} v\right)^{3}=0 \text {. }
\end{aligned}
$$


There are five equations to satisfy, namely,

$$
\begin{aligned}
2 c_{1} b_{1}^{4}+2 c_{2} b_{2}^{4}+2 c_{3} b_{3}^{4}+2 c_{4} b_{4}^{4} & =i, \\
-2 \frac{c_{1}}{b_{1}^{4}}-2 \frac{c_{2}}{b_{2}^{4}}-2 \frac{c_{3}}{b_{3}^{4}}-2 \frac{c_{4}}{b_{4}^{4}} & =i, \\
c_{1} b_{1}^{2}+c_{2} b_{2}^{2}+c_{3} b_{3}^{2}+c_{4} b_{4}^{2} & =0, \\
c_{1}+c_{2}+c_{3}+c_{4} & =0, \\
\frac{c_{1}}{b_{1}^{2}}+\frac{c_{2}}{b_{2}^{2}}+\frac{c_{3}}{b_{3}^{2}}+\frac{c_{4}}{b_{4}^{2}} & =0 .
\end{aligned}
$$

The constraints on the coefficients $b_{j}$ and $c_{j}$ can be written

$$
\begin{aligned}
& c_{1}=\frac{i b_{1}^{2}}{2\left(b_{1}^{2}-b_{2}^{2}\right)\left(b_{1}^{2}-b_{3}^{2}\right)\left(b_{1}^{2}-b_{4}^{2}\right)}, \\
& c_{2}=\frac{i b_{2}^{2}}{2\left(b_{2}^{2}-b_{1}^{2}\right)\left(b_{2}^{2}-b_{3}^{2}\right)\left(b_{2}^{2}-b_{4}^{2}\right)}, \\
& c_{3}=\frac{i b_{3}^{2}}{2\left(b_{3}^{2}-b_{1}^{2}\right)\left(b_{3}^{2}-b_{2}^{2}\right)\left(b_{3}^{2}-b_{4}^{2}\right)}, \\
& c_{4}=\frac{i b_{4}^{2}}{2\left(b_{4}^{2}-b_{1}^{2}\right)\left(b_{4}^{2}-b_{2}^{2}\right)\left(b_{4}^{2}-b_{3}^{2}\right)}, \\
& b_{1}= \pm \frac{1}{b_{2} b_{3} b_{4}} .
\end{aligned}
$$

For an octupole, there are three free parameters, which we can take to be $b_{2}, b_{3}$ and $b_{4}$.

\section{General multipole magnets}

The derivation of the constraints on the coefficients $c_{j}$ and $b_{j}$ in the case of quadrupoles, sextupoles and octupoles can be extended to any order of multipole. In the body of the multipole, the components of the field are

$$
\begin{aligned}
& B_{u}=i v^{n}, \\
& B_{v}=-i u^{n}, \\
& B_{\zeta}=0 .
\end{aligned}
$$

The falloff functions $F_{j}\left(\zeta+i h_{j}\right)$ and $G_{j}\left(\zeta-i h_{j}\right)$ satisfy for any $j$ :

$$
\begin{aligned}
& \lim _{\zeta \rightarrow-\infty} F_{j}\left(\zeta+i h_{j}\right)=1, \\
& \lim _{\zeta \rightarrow-\infty} G_{j}\left(\zeta-i h_{j}\right)=(-1)^{n+1},
\end{aligned}
$$

and

$$
\lim _{\zeta \rightarrow \infty} F_{j}\left(\zeta+i h_{j}\right)=\lim _{\zeta \rightarrow \infty} G_{j}\left(\zeta-i h_{j}\right)=0
$$

Substituting into (37), (38) and (39) gives

$$
\begin{array}{r}
i c_{1} b_{1}\left(i \frac{u}{b_{1}}+i b_{1} v+\zeta\right)^{n}+i c_{1} b_{1}\left(i \frac{u}{b_{1}}+i b_{1} v-\zeta\right)^{n}+i c_{2} b_{2}\left(i \frac{u}{b_{2}}+i b_{2} v+\zeta\right)^{n}+i c_{2} b_{2}\left(i \frac{u}{b_{2}}+i b_{2} v-\zeta\right)^{n} \\
+\cdots+i c_{n+1} b_{n+1}\left(i \frac{u}{b_{n+1}}+i b_{n+1} v+\zeta\right)^{n}+i c_{n+1} b_{n+1}\left(i \frac{u}{b_{n+1}}+i b_{n+1} v-\zeta\right)^{n}=i v^{n} \\
i \frac{c_{1}}{b_{1}}\left(i \frac{u}{b_{1}}+i b_{1} v+\zeta\right)^{n}+i \frac{c_{1}}{b_{1}}\left(i \frac{u}{b_{1}}+i b_{1} v-\zeta\right)^{n}+i \frac{c_{2}}{b_{2}}\left(i \frac{u}{b_{2}}+i b_{2} v+\zeta\right)^{n}+i \frac{c_{2}}{b_{2}}\left(i \frac{u}{b_{2}}+i b_{2} v-\zeta\right)^{n} \\
+\cdots+i \frac{c_{n+1}}{b_{n+1}}\left(i \frac{u}{b_{n+1}}+i b_{n+1} v+\zeta\right)^{n}+i \frac{c_{n+1}}{b_{n+1}}\left(i \frac{u}{b_{n+1}}+i b_{n+1} v-\zeta\right)^{n}=-i u^{n}, \\
c_{1}\left(i \frac{u}{b_{1}}+i b_{1} v+\zeta\right)^{n}-c_{1}\left(i \frac{u}{b_{1}}+i b_{1} v-\zeta\right)^{n}+c_{2}\left(i \frac{u}{b_{2}}+i b_{2} v+\zeta\right)^{n}-c_{2}\left(i \frac{u}{b_{2}}+i b_{2} v-\zeta\right)^{n} \\
+\cdots+c_{n+1}\left(i \frac{u}{b_{n+1}}+i b_{n+1} v+\zeta\right)^{n}-c_{n+1}\left(i \frac{u}{b_{n+1}}+i b_{n+1} v-\zeta\right)^{n}=0 .
\end{array}
$$

There are $n+2$ equations to satisfy. Two of the equations can be written

$$
\begin{aligned}
2 c_{1} b_{1}^{n+1}+2 c_{2} b_{2}^{n+1}+\cdots+2 c_{n+1} b_{n+1}^{n+1} & =(-i)^{n}, \\
-2 \frac{c_{1}}{b_{1}^{n+1}}-2 \frac{c_{2}}{b_{2}^{n+1}}-\cdots-2 \frac{c_{n+1}}{b_{n+1}^{n+1}} & =(-i)^{n} .
\end{aligned}
$$

The remaining equations have the form

$$
c_{1} b_{1}^{\alpha}+c_{2} b_{2}^{\alpha}+\cdots+c_{n+1} b_{n+1}^{\alpha}=0,
$$

where, for convenience, we have defined $\alpha=n-2 p$ with $p$ a positive integer. The number of equations is determined by the order of the multipole, and is expressed in the restriction that $-n<\alpha<n$. Solving the equations leads to the following constraints on the coefficients $b_{j}$ and $c_{j}$ : 


$$
\begin{aligned}
c_{1}= & \frac{(-i)^{n} b_{1}^{n-1}}{2 \prod_{j=2}^{n+1}\left(b_{1}^{2}-b_{j}^{2}\right)}, \\
c_{2}= & \frac{(-i)^{n} b_{2}^{n-1}}{2 \prod_{j=1, j \neq 2}^{n+1}\left(b_{2}^{2}-b_{j}^{2}\right)}, \\
& \vdots \\
c_{n}= & \frac{(-i)^{n} b_{n}^{n-1}}{2 \prod_{j=1, j \neq n}^{n+1}\left(b_{n}^{2}-b_{j}^{2}\right)}, \\
b_{1}= & \pm \frac{i^{n+1}}{\prod_{j=2}^{n+1} b_{j}} .
\end{aligned}
$$

With these relationships between the parameters, the field in a multipole magnet (including the fringe field region) is described by (37)-(39). The parameters $b_{j}$ for $j>1$ can be chosen arbitrarily. The dipole case is given by $n=0$; there are no free parameters, and the coefficients $c_{1}$ and $b_{1}$ are

$$
\begin{gathered}
c_{1}=\frac{1}{2 b_{1}}, \\
b_{1}= \pm i
\end{gathered}
$$

For any order multipole, apart from the constraints on the behavior of $F_{j}\left(\zeta+i h_{j}\right)$ and $G_{j}\left(\zeta-i h_{j}\right)$ for large negative and large positive values of $\zeta$, there is a great deal of freedom in choosing the functions $F_{j}\left(\zeta+i h_{j}\right)$ and $G_{j}\left(\zeta-i h_{j}\right)$ to best describe the field falloff in any particular case.

\section{E. Significance of the free parameters}

The solutions (37)-(39) with $F_{j}\left(\zeta+i h_{j}\right) \rightarrow 1$ and $G_{j}\left(\zeta-i h_{j}\right) \rightarrow(-1)^{n+1}$ as $\zeta \rightarrow-\infty$ describe magnets that have "pure" multipole fields within the body of the magnet (where the order of the multipole is specified by the value of $n$ ). In the fringe field region, because of the additional dependence on the transverse variables introduced by the functions $F_{j}\left(\zeta+i h_{j}\right)$ and $G_{j}\left(\zeta-i h_{j}\right)$, the field is not a pure multipole. As a consequence, if we evaluate the multipole gradient at a given longitudinal position in the fringe field region we will obtain a different value away from the axis of the magnet compared to the value we would obtain on the axis. This raises the possibility that, even when the falloff of the multipole gradient along the axis of a magnet is specified, there is some freedom to allow variation in the falloff of the multipole gradient away from the axis. This freedom is associated with the parameters $b_{j}$ (with $2 \leq j \leq n+1$ ).

As an example, consider a quadrupole magnet $(n=1)$ with an Enge-type falloff in the gradient along the axis: the appropriate forms for the functions $F_{j}\left(\zeta+i h_{j}\right)$ and $G_{j}\left(\zeta-i h_{j}\right)$ in this case will be given in Sec. V B. We can plot the quadrupole gradient $g_{1}$ defined by

$$
g_{1}=\frac{\partial B_{y}}{\partial x}=\frac{\partial B_{x}}{\partial y}
$$

as a function of $z$ for different values of $x$ and $y$, and for different values of $b_{2}$. An example is shown in Fig. 3 . The falloff of the quadrupole gradient along the axis of the magnet is determined by the form of the functions $F_{j}\left(\zeta+i h_{j}\right)$ and $G_{j}\left(\zeta-i h_{j}\right)$, and is independent of the parameter $b_{2}$ (since $h_{j}=0$ for $x=y=0$ ). Away from the axis of the magnet, the falloff of the gradient depends on the value of $b_{2}$ as well as on the values of $x$ and $y$.

In general, there will be singularities in the field that can be associated physically with sources of the magnetic field (e.g., the coil windings in an electromagnet). In Sec. V A we shall give an explicit expression for the locations of the singularities in the longitudinal field component in the case of a quadrupole with Enge-type falloff of the quadrupole gradient. We shall see that the transverse locations of the singularities depend on the value of the parameter $b_{2}$ : this is consistent with the parameter $b_{2}$ influencing the falloff of

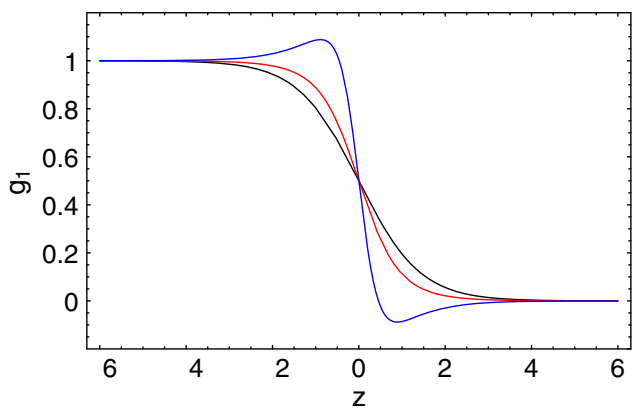

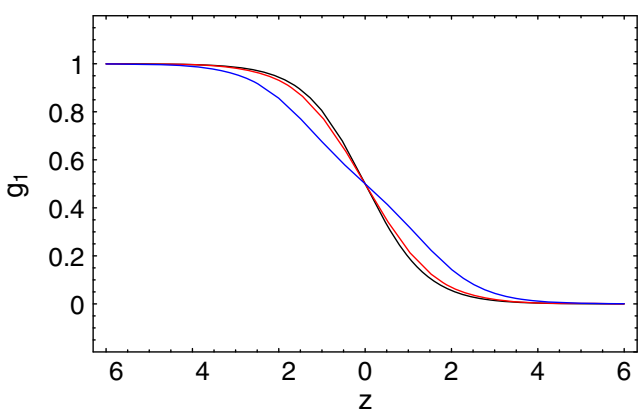

FIG. 3. Quadrupole gradient evaluated as a function of longitudinal position $z$ along the axis of the magnet (black line), and away from the axis for two different values of the parameter $b_{2}$ (red and blue lines). In the left-hand plot, the off-axis gradient is shown for $x=1 / \sqrt{2}, y=0$; in the right-hand plot, the off-axis gradient is shown for $x=0, y=1 / \sqrt{2}$. In each case, the red line shows the gradient for $b_{2}=2$, and the blue line shows the gradient for $b_{2}=4$. The gradient along the axis of the magnet is independent of the value of $b_{2}$. 
the gradient (as a function of longitudinal position) away from the magnetic axis.

We also observe in Fig. 3 that there is an asymmetry between the dependence of the gradient on the coordinates $x$ and $y$. That is, the falloff in the gradient (as a function of $z$ ) changes if we interchange $x$ and $y$. However, we see from (37)-(39) that in general, the dependence of the field on the transverse coordinates is contained in the quantities $h_{j}(40)$, given in Cartesian coordinates by

$$
h_{j}=\frac{1}{\sqrt{2}}\left(\frac{1}{b_{j}}+b_{j}\right) x+\frac{i}{\sqrt{2}}\left(\frac{1}{b_{j}}-b_{j}\right) y .
$$

Therefore, for an elementary solution (i.e., selecting a single $j$ ), changing the value of $b_{j}$ corresponds to rescaling the coordinates $x$ and $y$ (with some associated rescaling of the field). Furthermore, in the case of a quadrupole-type field $(n=1)$, replacing $b_{2}$ with $i / b_{2}$ corresponds to interchanging $x$ and $y$. Quadrupoles are often constructed with symmetric geometry so that $B_{y} \leftrightarrow B_{x}$ under $x \leftrightarrow y$. Although this symmetry does not appear in the expressions (37)-(39) for the full quadrupole solution (with $n=1$ and $m=2$ ), symmetry under interchange of the transverse coordinates can be imposed, if required, by appropriate superposition of full quadrupole solutions. This issue is discussed further in Sec. V B, but for now we simply stress that, although the symmetry $B_{y} \leftrightarrow B_{x}$ under $x \leftrightarrow y$ can be imposed if required, the solutions we have found for quadrupole fringe fields allow modeling of cases where this symmetry does not appear.

Finally, we note that the number of free parameters $b_{j}$ increases with increasing order $n$ of the multipole. This corresponds to additional degrees of freedom associated with the larger number of poles (and corresponding sources of the magnetic field) in multipoles of increasing order. Again, a symmetry on the field representing symmetry in the geometry of the magnet can be imposed if desired, but is not essential.

\section{MULTIPOLE FRINGE FIELDS WITH ENGE-TYPE FALLOFF}

Having constructed general expressions for fringe fields in multipole magnets, it is worth investigating these expressions further, to show that the field has the appropriate behavior. For a multipole magnet of arbitrary order, the expressions for the field can be rather complicated: therefore, we consider in detail only the case of a quadrupole. In this section, we shall discuss the behavior of the fields in a quadrupole, first for the elementary solution, and then for the full solution (in which the fields are given by real numbers) obtained by adding two versions of the elementary solution. In order to plot the field, we need to make some assumption for the form of the falloff of the gradient along the axis of the magnet: we shall consider the case that the falloff is described by an Enge function. At the end of the section, we shall generalize the expressions for a full quadrupole solution with Enge falloff of the gradient to higher order multipole magnets.

The appearance of singularities in the magnetic field is of some interest. Singularities are expected from the properties of Laplace's equation: in two dimensions, solutions to Laplace's equation that are bounded at infinity are either constant everywhere, or have singularities somewhere. Our expressions for multipole fringe fields have been obtained by extending the two-dimensional case to three dimensions. Mathematically, it is no surprise that singularities appear, but if the expressions we have derived for the fields are to be applied in physical situations, we should understand the position and nature of the singularities.

\section{A. Elementary solution for a quadrupole with Enge-type fringe field}

The full solution for a quadrupole can be constructed by combining elementary solutions with $n=1$. We shall consider in Sec. VB the full solution for a quadrupole in which the gradient falls off as an Enge function along the axis of the magnet; but to begin with, in the present section, we examine the behavior of the elementary solution with $n=1$. The field components are given by (31)-(33), with $f(\zeta+i h)$ and $g(\zeta-i h)$ given by (35) and (36). To produce an Enge-type falloff in the quadrupole gradient, the functions $F(\zeta+i h)$ and $G(\zeta-i h)$ must have an appropriate form. For simplicity we assume that $G(\xi)=F(\xi)$ for any (real or complex) argument $\xi$. The field components then become

$$
\begin{aligned}
B_{u} & =i b(\zeta+i h) F(\zeta+i h)-i b(\zeta-i h) F(\zeta-i h), \\
B_{v} & =\frac{i}{b}(\zeta+i h) F(\zeta+i h)-\frac{i}{b}(\zeta-i h) F(\zeta-i h), \\
B_{\zeta} & =(\zeta+i h) F(\zeta+i h)+(\zeta-i h) F(\zeta-i h) .
\end{aligned}
$$

Although the elementary solution does not represent a physical field, we can characterize the gradient in terms of the derivatives of the transverse components $B_{u}$ and $B_{v}$ with respect to $v$ and $u$ (respectively) along the $\zeta$ axis:

$$
\begin{aligned}
& \left.\frac{\partial B_{u}}{\partial v}\right|_{u=v=0}=-2 b^{2}\left[F(\zeta)+\zeta F^{\prime}(\zeta)\right], \\
& \left.\frac{\partial B_{v}}{\partial u}\right|_{u=v=0}=-\frac{2}{b^{2}}\left[F(\zeta)+\zeta F^{\prime}(\zeta)\right],
\end{aligned}
$$

where $F^{\prime}(\zeta)$ is the derivative of $F(\zeta)$ (with real argument $\zeta)$. We consider the case where the quadrupole gradient is described by a single-parameter Enge function, so that

$$
F(\zeta)+\zeta F^{\prime}(\zeta)=\frac{1}{1+e^{\zeta}} .
$$


This has solution

$$
F(\zeta)=1+\frac{\ln 2}{\zeta}-\frac{\ln \left(1+e^{\zeta}\right)}{\zeta},
$$

where we have chosen a constant of integration so that $F(\zeta)$ remains finite at $\zeta=0$.

The components of the field in a Cartesian basis can now be written:

$$
\begin{aligned}
& B_{x}=-\frac{\left(1+b^{2}\right)}{\sqrt{2} b}\left[2 h+i \ln \left(1+e^{\sqrt{2} z+i h}\right)-i \ln \left(1+e^{\sqrt{2} z-i h}\right)\right], \\
& B_{y}=-i \frac{\left(1-b^{2}\right)}{\sqrt{2} b}\left[2 h+i \ln \left(1+e^{\sqrt{2} z+i h}\right)-i \ln \left(1+e^{\sqrt{2} z-i h}\right)\right], \\
& B_{z}=\sqrt{2}\left[2 \sqrt{2} z+2 \ln 2-\ln \left(1+e^{\sqrt{2} z+i h}\right)-\ln \left(1+e^{\sqrt{2} z-i h}\right)\right],
\end{aligned}
$$

where, from (18), (19) and (34) the quantity $h$ is expressed in terms of the Cartesian coordinates:

$$
h=\frac{1}{\sqrt{2}}\left(\frac{1}{b}+b\right) x+\frac{i}{\sqrt{2}}\left(\frac{1}{b}-b\right) y .
$$

Along the axis of the magnet $(x=y=0)$, the transverse components of the field vanish and the longitudinal component of the field is purely real. The gradient of the field along the axis of the magnet is

$$
\left.\frac{\partial B_{y}}{\partial x}\right|_{x=y=0}=\left.\frac{\partial B_{x}}{\partial y}\right|_{x=y=0}=-i \frac{\left(1-b^{4}\right)}{b^{2}} \frac{1}{1+e^{\sqrt{2} z}} .
$$

This has the form of a single-parameter Enge function, as desired.

A physical magnetic field (with real components) can be constructed by adding together elementary solutions with appropriate coefficients; however, for now we identify the physical magnetic field with the imaginary parts of the elementary solution. In that case, the longitudinal component of the field vanishes along the axis. Singularities in the field occur when

$$
\sqrt{2} z \pm i h=i \ell \pi
$$

where $\ell$ is any odd integer. If $b$ is real, then in terms of the Cartesian coordinates, the singularities occur at

$$
(x, y)=\left(\frac{\sqrt{2} \ell \pi}{\frac{1}{b}+b}, \pm \frac{2 z}{\frac{1}{b}-b}\right) .
$$

Note, however, that the singularities in the different terms in the transverse components of the field cancel out when $z=0$.

The behavior of the field can be seen in Fig. 4, which shows the imaginary parts of the $B_{x}$ and $B_{z}$ components of the magnetic field as functions of the transverse coordinates at various $z$ locations. As noted in Sec. IV E, for an elementary solution a change in the constant $b_{j}$ amounts to a rescaling in the coordinates and the field; therefore, we show plots for only a single value $b=0.1$. Also, since no new features occur in the behavior of $B_{y}$ compared to the behavior of $B_{x}$, we only show plots for $B_{x}$ and $B_{z}$. The plots in Fig. 4 show that the field has the expected behavior for a quadrupole: in particular, within the body of the magnet, $B_{x}$ is linear in the coordinate $y$, and independent of $x$. At increasing values of $z$, the slope of $B_{x}$ versus $y$ decreases (the gradient falls off); at $z=0$, the quadrupole gradient is half the value at large negative $z$, by construction. As $z$ increases further, the gradient (and the field itself) falls to zero. The singularities in the field have the behavior expected from (48). At $z=0$, the singularities in the transverse field disappear completely, again, by construction.

Note that it was not necessary to demand that $G(\xi)=$ $F(\xi)$ in the falloff functions above. This was only done for simplicity and it could well be the case that a nonsymmetric falloff is desired, which would require $F(\xi)$ and $G(\xi)$ to be different from each other. As they are just functions that dictate the behavior of the field in the falloff (fringe) region, nothing changes inside the quadrupole and the magnetostatic Maxwell equations are still satisfied.

\section{B. Full solution for a quadrupole with Enge-type fringe field}

We can construct physical (real) fields in a quadrupole by adding two versions of the elementary solution with $n=1$. For simplicity, we assume that $F_{j}(\zeta)=G_{j}(\zeta)=F(\zeta)$ for all $j(=1,2)$. The field components are then written:

$B_{u}=\sum_{j=1}^{2} i b_{j} c_{j}\left[\left(\zeta+i h_{j}\right) F\left(\zeta+i h_{j}\right)-\left(\zeta-i h_{j}\right) F\left(\zeta-i h_{j}\right)\right]$,

$B_{v}=\sum_{j=1}^{2} i \frac{c_{j}}{b_{j}}\left[\left(\zeta+i h_{j}\right) F\left(\zeta+i h_{j}\right)-\left(\zeta-i h_{j}\right) F\left(\zeta-i h_{j}\right)\right]$,

$B_{\zeta}=\sum_{j=1}^{2} c_{j}\left[\left(\zeta+i h_{j}\right) F\left(\zeta+i h_{j}\right)+\left(\zeta-i h_{j}\right) F\left(\zeta-i h_{j}\right)\right]$,

where $h_{j}$ is given by (40) and the parameters $b_{j}$ and $c_{j}$ satisfy the relationships in (41)-(43).

The quadrupole gradient on the axis of the magnet $(u=v=0)$ is given by

$$
g(\zeta)=-\left.i \frac{\partial B_{u}}{\partial v}\right|_{u=v=0}=\left.i \frac{\partial B_{v}}{\partial u}\right|_{u=v=0}=F(\zeta)+\zeta F^{\prime}(\zeta) .
$$



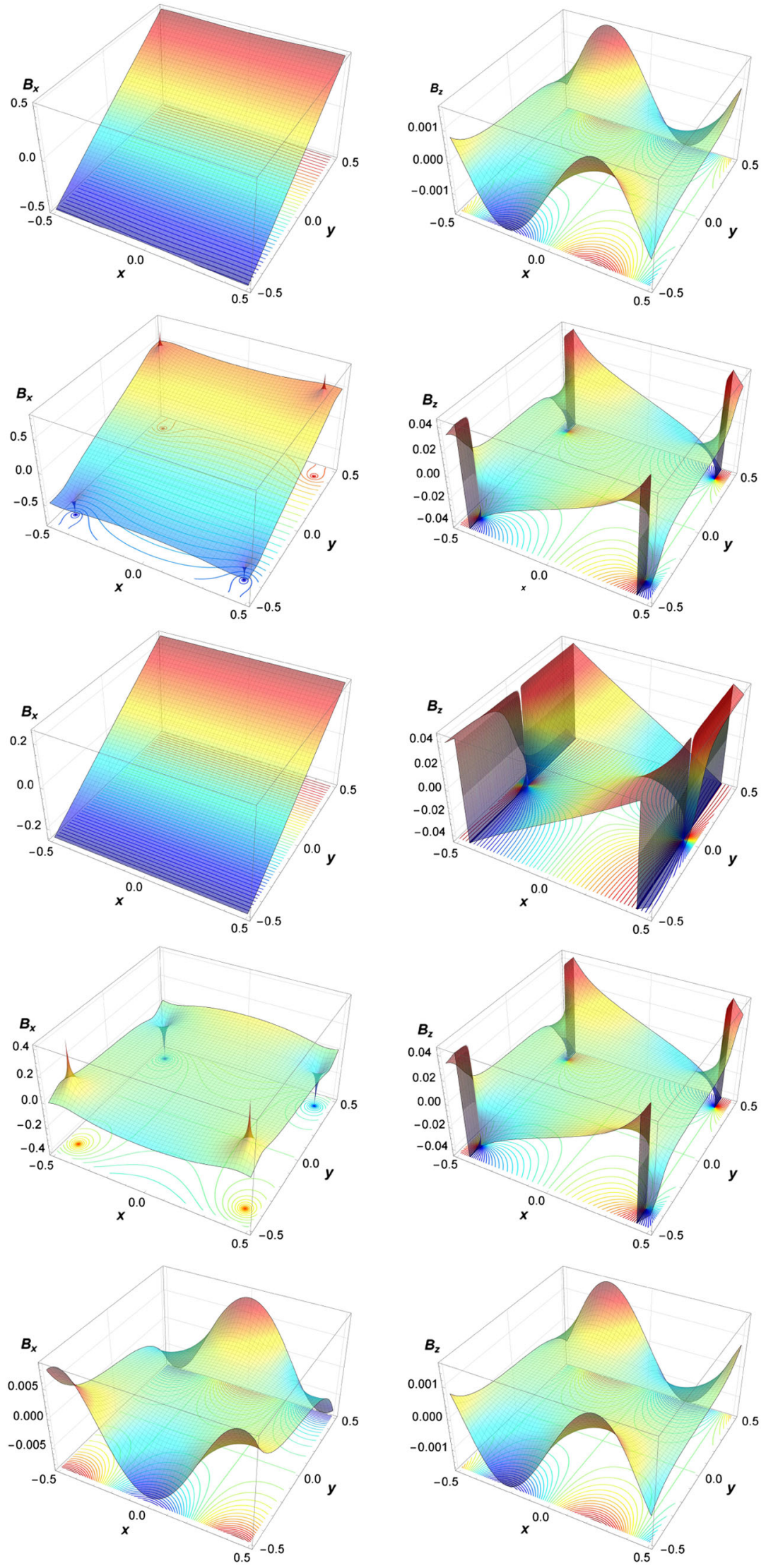

FIG. 4. Elementary quadrupole-like fringe field components $B_{x}$ (left) and $B_{z}$ (right) at (top to bottom) $z=-4.0, z=-2.0, z=0$, $z=2.0$ and $z=4.0$. 
A conventional model for the fringe field in a quadrupole magnet describes the gradient as an Enge function [18]. Again to keep the analysis as simple as possible, we consider the case that the gradient varies as a singleparameter Enge function, so that the function $F(\zeta)$ satisfies
(46). Writing the gradient in this way determines $\zeta=0$ as the location along the axis at which the quadrupole gradient falls to half its nominal value within the body of the magnet. Integrating (46) gives the expression (47) for the function $F(\zeta)$.

The components of the field can now be written (in Cartesian coordinates):

$$
\begin{gathered}
B_{x}=y+\frac{b}{2 \sqrt{2}\left(1-b^{2}\right)}\left[\ln \left(1+e^{\sqrt{2} z+i h}\right)-\ln \left(1+e^{\sqrt{2} z-i h}\right)+\ln \left(1+e^{\sqrt{2} z-i \bar{h}}\right)-\ln \left(1+e^{\sqrt{2} z+i \bar{h}}\right)\right], \\
B_{y}=x+\frac{i b}{2 \sqrt{2}\left(1+b^{2}\right)}\left[\ln \left(1+e^{\sqrt{2} z+i h}\right)-\ln \left(1+e^{\sqrt{2} z-i h}\right)-\ln \left(1+e^{\sqrt{2} z-i \bar{h}}\right)+\ln \left(1+e^{\sqrt{2} z+i \bar{h}}\right)\right], \\
B_{z}=-\frac{i b^{2}}{\sqrt{2}\left(1-b^{4}\right)}\left[\ln \left(1+e^{\sqrt{2} z+i h}\right)+\ln \left(1+e^{\sqrt{2} z-i h}\right)-\ln \left(1+e^{\sqrt{2} z-i \bar{h}}\right)-\ln \left(1+e^{\sqrt{2} z+i \bar{h}}\right)\right],
\end{gathered}
$$

where $b=b_{2}$ is a free parameter, $h=h_{2}$ (with $h_{2}$ given by (40) in the case that $j=2$ ), and $\bar{h}$ is given by

$$
\bar{h}=\frac{1}{\sqrt{2}}\left(\frac{1}{b}+b\right) x-\frac{i}{\sqrt{2}}\left(\frac{1}{b}-b\right) y .
$$

If $b$ is real, then $\bar{h}$ is the complex conjugate of $h$, and (since terms in the above expressions for the field components appear in complex conjugate pairs) the field components are purely real.

We observe that in the solution (52)-(54), the dependence of $B_{x}$ on the parameter $b$ is different from the dependence of $B_{y}$ on $b$. As a consequence, if we plot $B_{x}$ as a function of $z$ along a line $x=0, y=r_{0}$, we see a different variation compared to a plot of $B_{y}$ as a function of $z$ along a line $x=r_{0}, y=0$. In other words, as already noted in Sec. IV E, there is an asymmetry in the field in this case that would not be expected from a quadrupole magnet constructed with fourfold rotational symmetry about the $z$ axis. Such a magnet would generate a field with symmetry $B_{x} \leftrightarrow B_{y}$ under $x \leftrightarrow y$. Although the solution (52)-(54) does not display this symmetry, it can be imposed by adding equivalent expressions to $B_{x}, B_{y}$ and $B_{z}$, with $x$ and $y$ interchanged but keeping $b_{2}$ the same, and dividing the result by 2 . We refer to such a solution as a "full symmetrized solution." Figure 5 shows the behavior of the field components $B_{x}, B_{y}$ and $B_{z}$ for the full symmetrized quadrupole solution, with the fixed value of the parameter $b_{2}=0.1$. Within the body of the quadrupole (large negative $z$ ) we see that, as expected, $B_{x} \propto y, B_{y} \propto x$ and $B_{z} \approx 0$. At $z=0$ the quadrupole gradient falls to half of its value within the body of the magnet. At a large distance from the quadrupole (large positive z), the field approaches zero.

The full symmetrized solution has the interesting property that the field components remain finite in the limit $b \rightarrow 1$. The expressions for the field components then take a relatively simple form, although we lose a degree of freedom (provided by the parameter $b$ ) allowing some control over the variation of the fringe field with transverse position. However, the expressions may be useful in some cases, so it is worth stating the results:

$$
\begin{aligned}
& B_{x}=\left[3-\frac{\sinh (\sqrt{2} z)}{\cosh (\sqrt{2} z)+\cos (\sqrt{2} x)}\right] \frac{y}{4}-\frac{\sqrt{2}}{4} \arctan \left(\frac{\sin (\sqrt{2} y)}{e^{-\sqrt{2} z}+\cos (\sqrt{2} y)}\right), \\
& B_{y}=\left[3-\frac{\sinh (\sqrt{2} z)}{\cosh (\sqrt{2} z)+\cos (\sqrt{2} y)}\right] \frac{x}{4}-\frac{\sqrt{2}}{4} \arctan \left(\frac{\sin (\sqrt{2} x)}{e^{-\sqrt{2} z}+\cos (\sqrt{2} x)}\right), \\
& B_{z}=-\left[\frac{\sin (\sqrt{2} y)}{\cosh (\sqrt{2} z)+\cos (\sqrt{2} y)}\right] \frac{x}{4}-\left[\frac{\sin (\sqrt{2} x)}{\cosh (\sqrt{2} z)+\cos (\sqrt{2} x)}\right] \frac{y}{4} .
\end{aligned}
$$



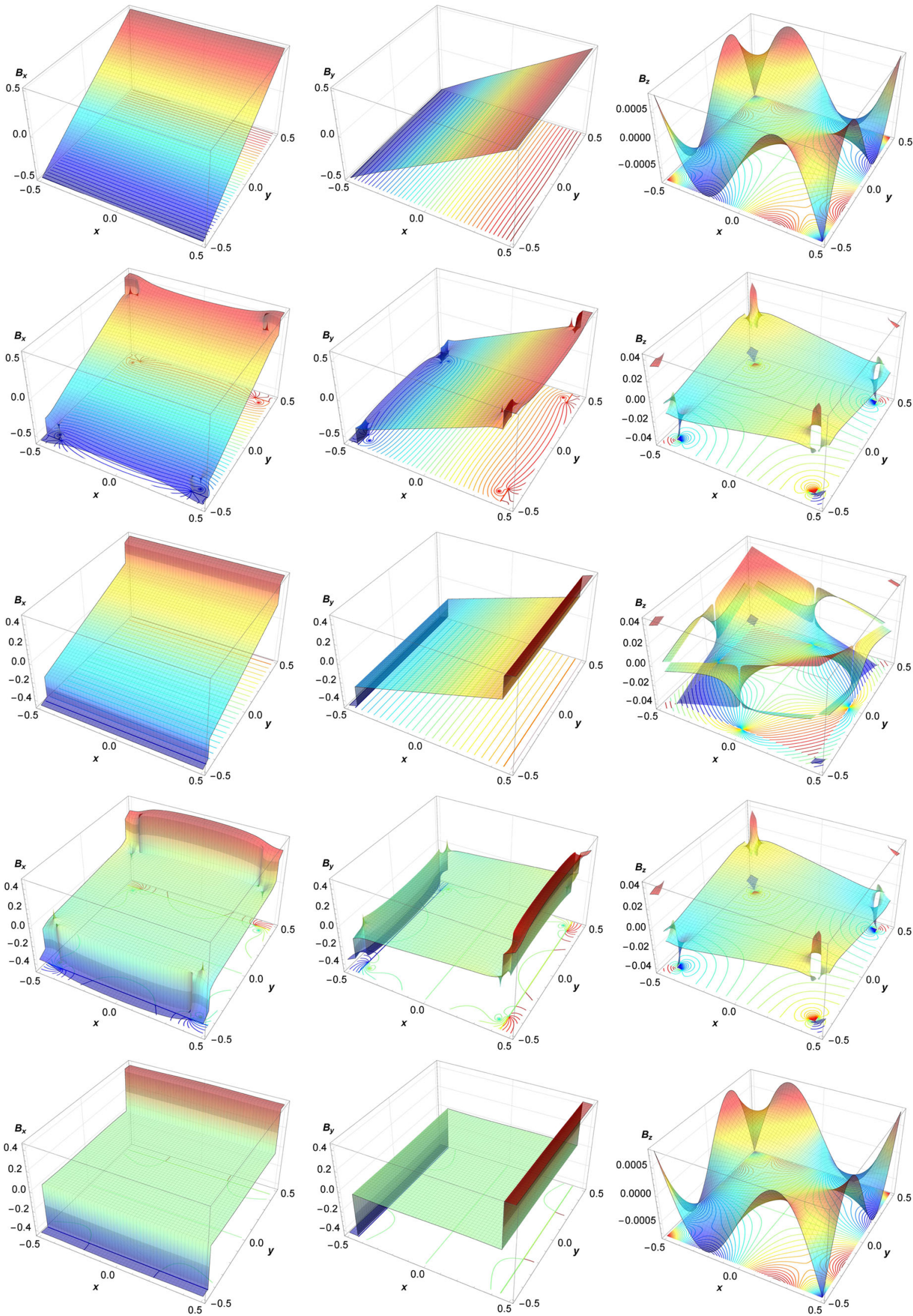

FIG. 5. Quadrupole fringe field components $B_{x}$ (left), $B_{y}$ (center) and $B_{z}$ (right) at (top to bottom) $z=-4.0, z=-2.0, z=0, z=2.0$ and $z=4.0$. 


\section{Full solution for a multipole magnet with Enge-type fringe field}

Using (37)-(39), the full solution for a multipole of order $n$ with Enge-type falloff of the gradient can be written:

$$
\begin{aligned}
B_{u} & =\sum_{j=1}^{n+1} i b_{j} c_{j}\left[\left(\zeta+i h_{j}\right)^{n} F\left(n ; \zeta+i h_{j}\right)-(-1)^{n+1}\left(\zeta-i h_{j}\right)^{n} F\left(n ; \zeta-i h_{j}\right)\right], \\
B_{v} & =\sum_{j=1}^{n+1} i \frac{c_{j}}{b_{j}}\left[\left(\zeta+i h_{j}\right)^{n} F\left(n ; \zeta+i h_{j}\right)-(-1)^{n+1}\left(\zeta-i h_{j}\right)^{n} F\left(n ; \zeta-i h_{j}\right)\right], \\
B_{\zeta} & =\sum_{j=1}^{n+1} c_{j}\left[\left(\zeta+i h_{j}\right)^{n} F\left(n ; \zeta+i h_{j}\right)+(-1)^{n+1}\left(\zeta-i h_{j}\right)^{n} F\left(n ; \zeta-i h_{j}\right)\right],
\end{aligned}
$$

where the parameters $b_{j}$ and $c_{j}$ satisfy the relationships (41)-(43), and we have chosen for all $j$ :

$$
\begin{aligned}
& F_{j}\left(\zeta+i h_{j}\right)=F\left(n ; \zeta+i h_{j}\right), \\
& G_{j}\left(\zeta-i h_{j}\right)=(-1)^{n+1} F\left(n ; \zeta-i h_{j}\right) .
\end{aligned}
$$

The function $F(n ; \xi)$ with complex argument $\xi$ is constructed so that the multipole gradient has an Enge-type falloff in the fringe field region. This is achieved if

$$
F(0 ; \xi)=\frac{1}{1+e^{\xi}},
$$

and the functions $F(n ; \xi)$ for $n>0$ are then obtained by induction, as follows. We define (for real $\zeta$ )

$$
\frac{\zeta^{n}}{n !} F(n ; \zeta)=\int_{0}^{\zeta} \frac{\zeta^{\prime n-1}}{(n-1) !} F\left(n-1 ; \zeta^{\prime}\right) \mathrm{d} \zeta^{\prime} .
$$

For any (positive integer) $n$ and (real argument) $\zeta, F(n ; \zeta)$ can be written in the form

$F(n ; \zeta)=1+\frac{n !}{\zeta^{n}} \mathrm{Li}_{n}\left(-e^{\zeta}\right)-\sum_{j=1}^{n} \frac{n !}{(n-j) ! \zeta^{j}} \mathrm{Li}_{j}(-1)$

where $\operatorname{Li}_{n}(\zeta)$ is the polylogarithm (or Jonquière function [19]) of order $n$. The functions $F(n ; \zeta)$ have the limits $F(n ; \zeta) \rightarrow 1$ as $\zeta \rightarrow-\infty$, and $F(n ; \zeta) \rightarrow 0$ as $\zeta \rightarrow \infty$. The domain of $F(n ; \zeta)$ is extended to the complex plane by substituting a complex variable $\xi$ for $\zeta$ in (57). The multipole gradient on the magnetic axis $(u=v=0)$ is given by

$$
\left.\frac{-i}{n !} \frac{\partial^{n} B_{u}}{\partial v^{n}}\right|_{u=v=0}=\left.\frac{i}{n !} \frac{\partial^{n} B_{v}}{\partial u^{n}}\right|_{u=v=0}=\frac{1}{1+e^{\zeta}},
$$

which is a single-parameter Enge function, as desired.

\section{POTENTIALS FOR MULTIPOLE MAGNET FRINGE FIELDS}

For some applications it may be useful to have analytical expressions for scalar and vector potentials from which the fringe fields in multipole magnets can be derived. For example, in an iron dominated magnet the pole faces correspond to surfaces of constant scalar potential; this makes it possible to inspect the geometry of a magnet that would have a fringe field of a given form. The vector potential can be useful for symplectic tracking of particles through the field (see, for example, [20]). In this section, we state expressions for the scalar and vector potentials for multipole magnets with arbitrary falloff of the gradient in the fringe field region; we then consider the particular case that the multipole gradient has a falloff that can be represented by an Enge function.

\section{A. Scalar potential}

The magnetic scalar potential $\varphi=\varphi(x, y, z)$ is defined so that

$$
\vec{B}=\nabla \varphi .
$$

In terms of coordinates $u, v, \zeta$, this can be written

$$
\left(B_{u}, B_{v}, B_{\zeta}\right)=\left(\frac{\partial \varphi}{\partial v}, \frac{\partial \varphi}{\partial u}, \frac{\partial \varphi}{\partial \zeta}\right) .
$$

The elementary solution (31)-(33) can be derived from the potential

$$
\varphi=\tilde{f}(\zeta+i h)+\tilde{g}(\zeta-i h),
$$

where the functions $\tilde{f}(\zeta)$ and $\tilde{g}(\zeta)$ for real argument $\zeta$ are the integrals of $f(\zeta)$ and $g(\zeta)$ :

$$
\begin{aligned}
& \tilde{f}(\zeta)=\int_{0}^{\zeta} f\left(\zeta^{\prime}\right) \mathrm{d} \zeta^{\prime}, \\
& \tilde{g}(\zeta)=\int_{0}^{\zeta} g\left(\zeta^{\prime}\right) \mathrm{d} \zeta^{\prime} .
\end{aligned}
$$


The integrals are performed on the real axis, and the functions thus obtained are then extended to the complex plane simply by substitution of a complex argument.

As an example, in the case of a multipole field (of order $n$ ) with an Enge-type falloff in the gradient, the function $f(\zeta)$ is

$$
f(\zeta)=\zeta^{n} F(n ; \zeta)
$$

where $F(n ; \zeta)$ is given by (57). Using (56), and replacing the real variable $\zeta$ by a complex variable $\xi$, we then find that

$$
\tilde{f}(\xi)=\frac{\xi^{n+1}}{n+1} F(n+1 ; \xi)
$$

Similarly

$$
\tilde{g}(\xi)=(-1)^{n+1} \frac{\xi^{n+1}}{n+1} F(n+1 ; \xi)
$$

The scalar potential is therefore given by

$$
\begin{aligned}
\varphi= & \frac{1}{n+1} \sum_{j=1}^{n+1} c_{j}\left[\left(\zeta+i h_{j}\right)^{n+1} F\left(n+1 ; \zeta+i h_{j}\right)\right. \\
& \left.+(-1)^{n+1}\left(\zeta-i h_{j}\right)^{n+1} F\left(n+1 ; \zeta-i h_{j}\right)\right],
\end{aligned}
$$

where the coefficients $c_{j}$ and $b_{j}$ (implicit in $h_{j}$ ) satisfy (41)-(43).

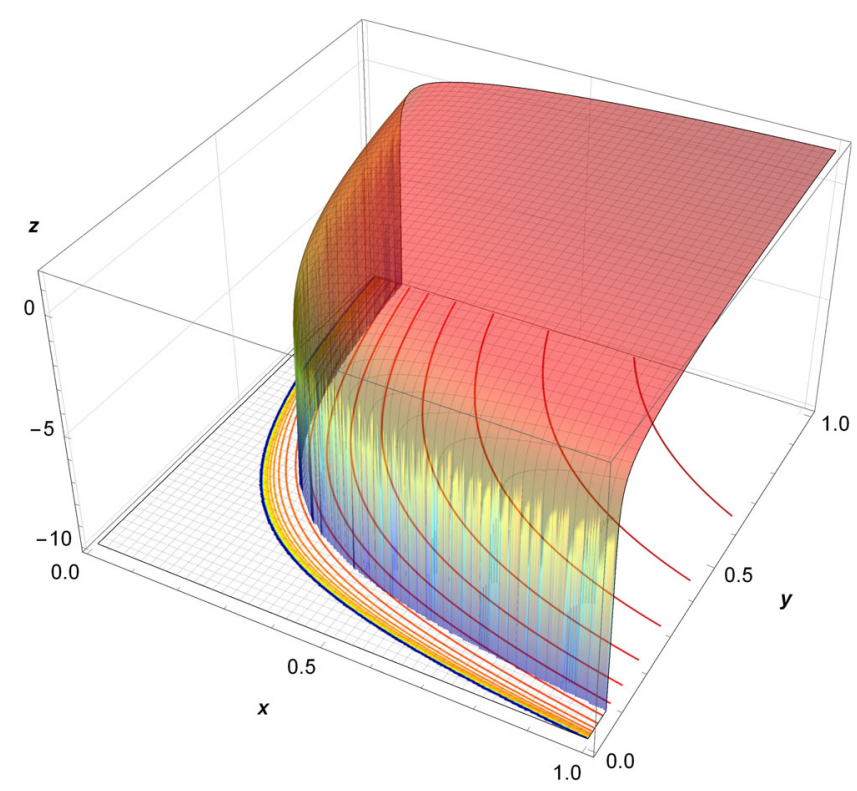

FIG. 6. Surface of constant scalar potential in a representation of the HL-LHC inner triplet quadrupole, with the gradient falling as an Enge function in the fringe field. The overall length scale is arbitrary. The parameters of the Enge function (63) correspond to the first line in Table I.
In the case of a dipole $(n=0)$ with Enge-type falloff of the field in the plane $y=0$, the on-axis field is

$$
\left.B_{y}\right|_{y=0}=\frac{\sqrt{2}}{1+e^{\sqrt{2} z}},
$$

where the factor $\sqrt{2}$ in the numerator ensures consistency with (28)-(30). Putting $n=0$ in (61), and using (44) and (45) for $c_{1}$ and $b_{1}$, we find

$$
\varphi=\sqrt{2} y+\frac{i}{2} \ln \left(1+e^{\sqrt{2}(z+i y)}\right)-\frac{i}{2} \ln \left(1+e^{\sqrt{2}(z-i y)}\right),
$$

which corresponds to (14) with suitable rescaling of the coordinates and field strength. The field obtained is then

$$
\begin{aligned}
& B_{x}=0 \\
& B_{y}=\frac{\sqrt{2}\left(1+e^{\sqrt{2} z} \cos (\sqrt{2} y)\right)}{1+2 e^{\sqrt{2} z} \cos (\sqrt{2} y)+e^{2 \sqrt{2} z}}, \\
& B_{z}=-\frac{\sqrt{2} e^{\sqrt{2} z} \sin (\sqrt{2} y)}{1+2 e^{\sqrt{2} z} \cos (\sqrt{2} y)+e^{2 \sqrt{2} z}},
\end{aligned}
$$

in agreement with (11) and (12) (again with suitable rescaling of the coordinates and field strength).

For the particular case of a quadrupole $(n=1)$ with Enge-type falloff of the gradient, the scalar potential can be written in Cartesian coordinates:

$$
\begin{aligned}
\varphi= & x y+\frac{i b^{2}}{2\left(1-b^{4}\right)}\left[\operatorname{Li}_{2}\left(-e^{\sqrt{2} z+i h}\right)+\operatorname{Li}_{2}\left(-e^{\sqrt{2} z-i h}\right)\right. \\
& \left.-\mathrm{Li}_{2}\left(-e^{\sqrt{2} z-i \bar{h}}\right)-\mathrm{Li}_{2}\left(-e^{\sqrt{2} z+i \bar{h}}\right)\right],
\end{aligned}
$$

where $b=b_{2}$ is an arbitrary parameter, and $h=h_{2}$.

In an iron dominated magnet, the pole faces can be identified with surfaces of constant scalar potential: an example of such a surface (based on an inner triplet quadrupole for the HL-LHC) is shown in Fig. 6. The surface has the geometry expected of the poles in an iron dominated quadrupole. Within the body of the magnet, the intersection of the pole face with a plane of constant $z$ forms a hyperbola. As a function of position along the axis, the pole face has little dependence on $z$ for large negative $z$; but in the fringe field region the pole face abruptly "flattens" off to lie close to the plane $z=0$.

It is worth noting that the form for the scalar potential (58) for the elementary solution provides a direct connection with Whittaker's form (17) for solutions to Laplace's equation in three dimensions. In Cartesian coordinates, the potential for the elementary solution can be written

$$
\varphi=\tilde{f}(\sqrt{2} z+i h)+\tilde{g}(\sqrt{2} z-i h)
$$


where, using (18), (19) and (34),

$$
h=\frac{1}{\sqrt{2}}\left(\frac{1}{b}+b\right) x+\frac{i}{\sqrt{2}}\left(\frac{1}{b}-b\right) y .
$$

If we define a parameter $\vartheta$ such that

$$
b=e^{-i \vartheta},
$$

then we can write

$$
h=\sqrt{2} x \cos \vartheta+\sqrt{2} y \sin \vartheta,
$$

and the potential for the elementary solution is

$$
\begin{aligned}
\varphi=\tilde{f} & {[\sqrt{2}(z+i x \cos \vartheta+i y \sin \vartheta)] } \\
& +\tilde{g}[\sqrt{2}(z-i x \cos \vartheta-i y \sin \vartheta)],
\end{aligned}
$$

which is in the form of a sum of two of Whittaker's solutions (17) (to obtain the second term, we need to replace $\vartheta$ by $\vartheta+\pi$ in Whittaker's solution). In Whittaker's form, it is natural to take $\vartheta$ as a real parameter, which corresponds to a complex value for $b$ (lying on the unit circle). In general, however, it is possible for $\vartheta$ to take any complex value. In the expressions presented in this paper, on the other hand, it is natural to take real values for the parameter $b$ (corresponding to $b_{2}$ for quadrupoles; for higher-order multipoles there are additional parameters, corresponding to additional terms in Whittaker's solution). However, it is of course possible to choose complex values for $b$. In the examples presented in Sec. VII, we find it convenient when fitting "real" quadrupole fringe fields to choose real values for $b$ lying off the unit circle.

\section{B. Vector potential}

It can be useful to know the vector potential for a given field for symplectic integration of the equations of motion for a charged particle moving through the field: see, for example, [20]. The field $\vec{B}$ is derived from the vector potential $\vec{A}$ by the usual relationship:

$$
\vec{B}=\nabla \times \vec{A} .
$$

It is possible to make a choice of gauge in which any one of the components of $\vec{A}$ vanishes: we choose to work in a gauge in which $A_{z}=0$. In the coordinates $u, v, \zeta$, this gauge choice is expressed as $A_{\zeta}=0$, and the field components are obtained from the vector potential by

$$
\begin{aligned}
& B_{u}=\sqrt{2} i \frac{\partial A_{u}}{\partial \zeta} \\
& B_{v}=-\sqrt{2} i \frac{\partial A_{v}}{\partial \zeta} \\
& B_{\zeta}=\frac{1}{\sqrt{2} i}\left(\frac{\partial A_{u}}{\partial u}-\frac{\partial A_{v}}{\partial v}\right) .
\end{aligned}
$$

For a field described by the elementary solution (31)-(33), it is readily verified that the vector potential is given by

$$
\begin{aligned}
& A_{u}=\frac{b}{\sqrt{2}}[\tilde{f}(\zeta+i h)-\tilde{g}(\zeta-i h)], \\
& A_{v}=-\frac{1}{\sqrt{2} b}[\tilde{f}(\zeta+i h)-\tilde{g}(\zeta-i h)], \\
& A_{\zeta}=0,
\end{aligned}
$$

where, as before, $\tilde{f}(\xi)$ and $\tilde{g}(\xi)$ are the integrals of $f(\xi)$ and $g(\xi)$. This potential satisfies the Coulomb gauge condition:

$$
\nabla \cdot \vec{A}=\frac{\partial A_{u}}{\partial u}+\frac{\partial A_{v}}{\partial v}+\frac{\partial A_{\zeta}}{\partial \zeta}=0 .
$$

For the particular case of a multipole magnet with Engetype falloff of the gradient, the functions $\tilde{f}(\xi)$ and $\tilde{g}(\xi)$ are the same as in the case of the scalar potential, (59) and (60). The vector potential is then

$$
\begin{aligned}
& A_{u}=\frac{1}{n+1} \sum_{j=1}^{n+1} \frac{c_{j} b_{j}}{\sqrt{2}}\left[\left(\zeta+i h_{j}\right)^{n+1} F\left(n+1 ; \zeta+i h_{j}\right)-(-1)^{n+1}\left(\zeta-i h_{j}\right)^{n+1} F\left(n+1 ; \zeta-i h_{j}\right)\right], \\
& A_{v}=-\frac{1}{n+1} \sum_{j=1}^{n+1} \frac{c_{j}}{\sqrt{2} b_{j}}\left[\left(\zeta+i h_{j}\right)^{n+1} F\left(n+1 ; \zeta+i h_{j}\right)-(-1)^{n+1}\left(\zeta-i h_{j}\right)^{n+1} F\left(n+1 ; \zeta-i h_{j}\right)\right], \\
& A_{\zeta}=0,
\end{aligned}
$$

where (as usual) the coefficients $c_{j}$ and $b_{j}$ satisfy (41)-(43).

For a dipole the vector potential is obtained with $n=0$, and with $c_{1}$ and $b_{1}$ given by (44) and (45) respectively:

$$
\begin{aligned}
& A_{x}=\sqrt{2} z-\frac{1}{2} \ln \left(1+e^{\sqrt{2}(z+i y)}\right)-\frac{1}{2} \ln \left(1+e^{\sqrt{2}(z-i y)}\right)+\ln 2, \\
& A_{y}=0 \\
& A_{z}=0
\end{aligned}
$$


in agreement with (16) with suitable rescaling of the coordinates and field strength (and with an additive constant, that vanishes when taking the curl of the vector potential).

For a quadrupole $(n=1)$, the vector potential can be expressed in Cartesian coordinates as

$$
\begin{aligned}
& A_{x}=x z+\frac{\ln 2}{\sqrt{2}} x-\frac{i b}{4\left(1+b^{2}\right)}\left[\operatorname{Li}_{2}\left(-e^{\sqrt{2} z+i h}\right)-\mathrm{Li}_{2}\left(-e^{\sqrt{2} z-i h}\right)-\mathrm{Li}_{2}\left(-e^{\sqrt{2} z-i \bar{h}}\right)+\mathrm{Li}_{2}\left(-e^{\sqrt{2} z+i \bar{h}}\right)\right], \\
& A_{y}=-y z-\frac{\ln 2}{\sqrt{2}} y+\frac{b}{4\left(1-b^{2}\right)}\left[\operatorname{Li}_{2}\left(-e^{\sqrt{2} z+i h}\right)-\operatorname{Li}_{2}\left(-e^{\sqrt{2} z-i h}\right)+\mathrm{Li}_{2}\left(-e^{\sqrt{2} z-i \bar{h}}\right)-\operatorname{Li}_{2}\left(-e^{\sqrt{2} z+i \bar{h}}\right)\right], \\
& A_{z}=0,
\end{aligned}
$$

where $b=b_{2}$ is an arbitrary parameter, and $h=h_{2}$.

\section{EXAMPLES}

To illustrate the application of the methods and results described in the previous sections, we consider two examples: a quadrupole in a final focus or "inner triplet" region of the high luminosity upgrade of the Large Hadron Collider (HL-LHC), and a quadrupole in the EMMA nonscaling fixed-field alternating gradient accelerator. These magnets are chosen to provide two contrasting cases in terms of magnet technology and parameter regime. The HL-LHC inner triplet quadrupole is a superconducting magnet with large aperture and gradient (respectively, $150 \mathrm{~mm}$ diameter and $140 \mathrm{~T} / \mathrm{m}$ ). Design studies are still in progress; some field maps are available, but the impact of the fringe fields on the beam dynamics are still under investigation. The EMMA quadrupoles are normalconducting electromagnets with more conventional gradient. Here, we consider one of the two types of EMMA magnets, namely the F quadrupoles [21]. These magnets have large apertures (74 $\mathrm{mm}$ diameter) given the length of the iron poles $(73 \mathrm{~mm})$; as a result, the fringe fields make a dominant contribution to the focusing effects. The specified integrated gradient is $0.387 \mathrm{~T}$. Although it is possible to represent an EMMA quadrupole by a hard-edge model, beam dynamics studies (supported by experimental results) [22] indicate the need for a more realistic representation in order to give an accurate description of the longitudinal and transverse dynamics in the machine.

We should emphasize that the purpose of considering these illustrative cases is not to demonstrate close agreement between the numerical and analytical field models, but simply to show that it is possible, using analytical formulas from the previous sections, to construct a model of the fringe field in each case that satisfies Maxwell's equations, and is closer to reality than a simple hard-edge model.

For the HL-LHC inner triplet quadrupole and for the EMMA $\mathrm{F}$ quadrupole we use the full solution for a quadrupole with Enge-type fringe field, presented in Sec. V B. The numerical field data in each case are fitted using a falloff function such that the quadrupole gradient has the form of an Enge function:

$$
g(z)=\frac{a_{0}}{1+e^{a_{1}+\sqrt{2} a_{2} z}} .
$$

The fit is optimized by varying the parameters $a_{0}, a_{1}$ and $a_{2}$. It is straightforward to generalize the results of Sec. V B to include the presence of additional parameters [as compared to the simplified form given in (46)]. Note that we use a full symmetrized solution for the quadrupole field, so that the symmetry $B_{y} \leftrightarrow B_{x}$ under $x \leftrightarrow y$ is enforced by superposition of appropriate full quadrupole solutions.

\section{A. HL-LHC inner triplet quadrupoles}

As a first example, we show the results of a fit to the field in an HL-LHC inner triplet quadrupole. Field data are obtained from a magnetic model, and the parameters $a_{0}, a_{1}$ and $a_{2}$ describing the gradient (63) are then adjusted using a nonlinear fitting routine (with some chosen value for the free parameter $b_{2}$ : see the comments below). The fit is performed to the radial field component along a line $x=y=r / \sqrt{2}$, where $r$ is the distance of the line from the axis of the magnet. If the model provides a good description of the field data, then the parameters obtained from the fit should be independent of the value of $r$ : results for some selected values of $r$ are shown in Table I. For the HL-LHC inner triplet quadrupole, inspection of the values in Table I shows changes in the fit parameters of less than $0.7 \%$, comparing values obtained by fitting the field along lines with $r=r_{\max } / 10$ and $r=r_{\max } / 6$ (where

TABLE I. Parameters for a fit of an Enge function of the form (63), to the quadrupole gradient in an HL-LHC inner triplet quadrupole. The maximum radius $r_{\max }$ in the field data is $75 \mathrm{~mm}$.

\begin{tabular}{lccc}
\hline \hline Radius of fit & $a_{0}$ & $a_{1}$ & $a_{2}$ \\
\hline$r_{\max } / 10$ & -55.9503 & -0.520120 & 8.98913 \\
$r_{\max } / 8$ & -55.9504 & -0.521262 & 9.00549 \\
$r_{\max } / 6$ & -55.9505 & -0.523743 & 9.04082 \\
$r_{\max } / 4$ & -55.9510 & -0.530875 & 9.14139 \\
$r_{\max } / 3$ & -55.9517 & -0.540984 & 9.28116 \\
$r_{\max } / 2$ & -55.9539 & -0.570142 & 9.66955 \\
\hline \hline
\end{tabular}




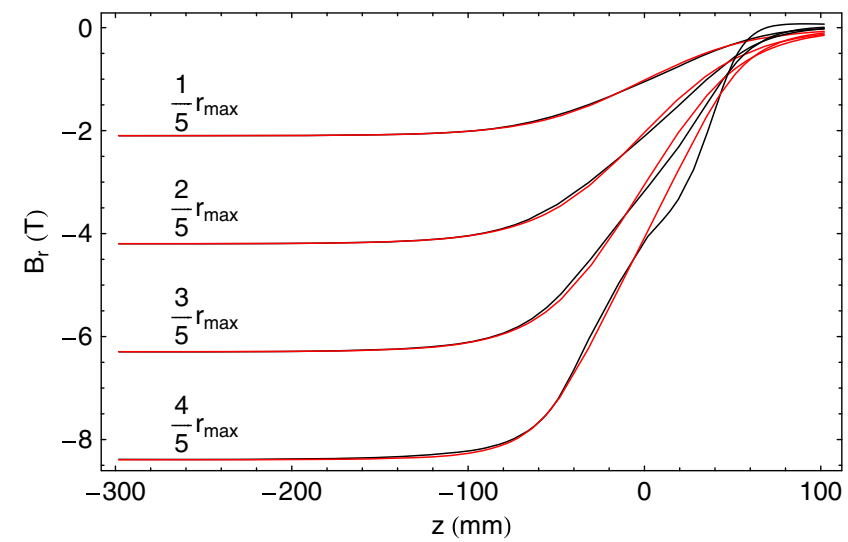

FIG. 7. Radial component of the magnetic field in an HL-LHC inner triplet quadrupole as a function of position along lines of given radius and with cylindrical polar coordinate $\theta=\pi / 4$. Each pair of black and red lines shows the field at a different radius, from $r_{\max } / 5$ to $4 r_{\max } / 5$, with $r_{\max }=75 \mathrm{~mm}$. The black lines show the field obtained from the (numerical) magnetic model; the red lines show a symmetrized form of the analytical model (49)-(51), with fit parameters given in Table I with radius of fit $r_{\max } / 10$, and $b_{2}=2.5$.

$r_{\max }=75 \mathrm{~mm}$ is the maximum radius for which field data are available).

Having obtained values for the fit parameters, we can make direct comparisons of the field based on Eqs. (49)-(51) with the field obtained by the numerical magnetic model. An example of such a comparison, for the radial field component, is shown in Fig. 7. Although the analytical model does not match the numerical model exactly, the general behavior of the field is reproduced quite closely. The impact of the residuals from the fit on the beam dynamics still needs to be studied; but the analytical model does include features of the field that would be completely omitted in a hard-edged magnet model.

Figure 8 shows the radial field component as a function of position along lines of fixed radius and for different values of the polar coordinate $\theta$. Again, there is reasonable agreement between the numerical field data (black lines) and the analytical fit (red line). Finally, Fig. 9 shows the longitudinal field component as a function of position along lines of given radius and with fixed $\theta=\pi / 4$. Here, it appears that there are more significant discrepancies between the numerical field data and the analytical model; however, there is general agreement in the main features, especially for the region close to the axis of the magnet. The more detailed structure that appears at larger distances from the axis cannot be reproduced by the relatively simple (Enge) function that is used to describe the falloff of the quadrupole gradient in the fringe field.

It is worth considering the dependence of the field on the parameter $b_{2}$. Changing the value of $b_{2}$ has an effect on the way that the fringe field varies with distance from the magnetic axis. This can be seen by comparing the plots in

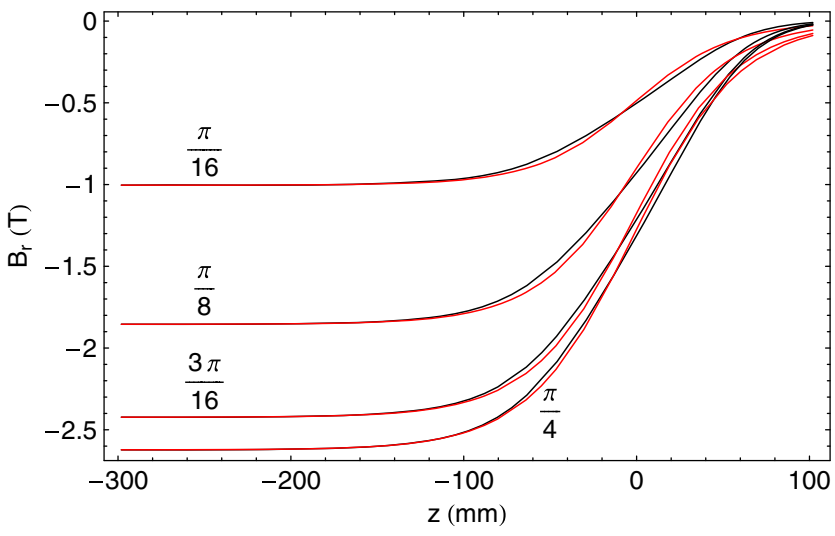

FIG. 8. Radial component of the magnetic field in an HL-LHC inner triplet quadrupole as a function of position along lines of fixed distance $r=r_{\max } / 4$. Each pair of black and red lines shows the field at a different value of the cylindrical polar coordinate $\theta$, from $\pi / 16$ to $\pi / 4$ (in steps of $\pi / 16$ ). The black lines show the field obtained from the (numerical) magnetic model; the red lines show a symmetrized form of the analytical model (49)-(51), with fit parameters given in Table I with radius of fit $r_{\max } / 10$, and $b_{2}=2.5$.

Fig. 10, which show the radial field component as a function of position along lines with fixed polar coordinate $\theta=\pi / 4$ and different distance $r$ from the axis of the magnet. The plot on the left in Fig. 10 shows a comparison between the numerical field map and the analytical model with $b_{2}=1.5$; the plot on the right compares the numerical field map and the analytical model with $b_{2}=3.5$. In regions close to the axis of the magnet, changes in the value of $b_{2}$ in the range 1.5 to 3.5 have little effect on the

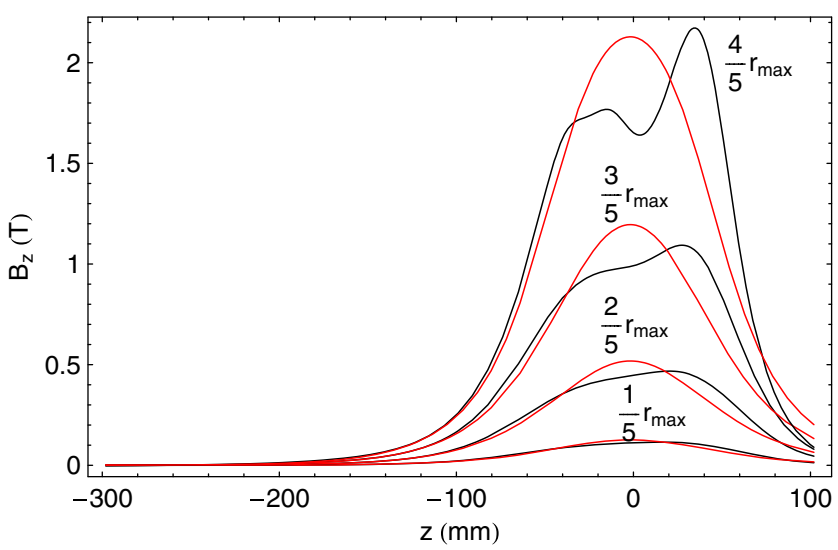

FIG. 9. Longitudinal component of the magnetic field in an HL-LHC inner triplet quadrupole as a function of position along lines of given radius and with cylindrical polar coordinate $\theta=\pi / 4$. Each pair of black and red lines shows the field at an increasing radius, from $r_{\max } / 5$ to $4 r_{\max } / 5$, with $r_{\max }=75 \mathrm{~mm}$. The black lines show the field obtained from the (numerical) magnetic model; the red lines show a symmetrized form of the analytical model (49)-(51), with fit parameters given in Table I with radius of fit $r_{\max } / 10$, and $b_{2}=2.5$. 

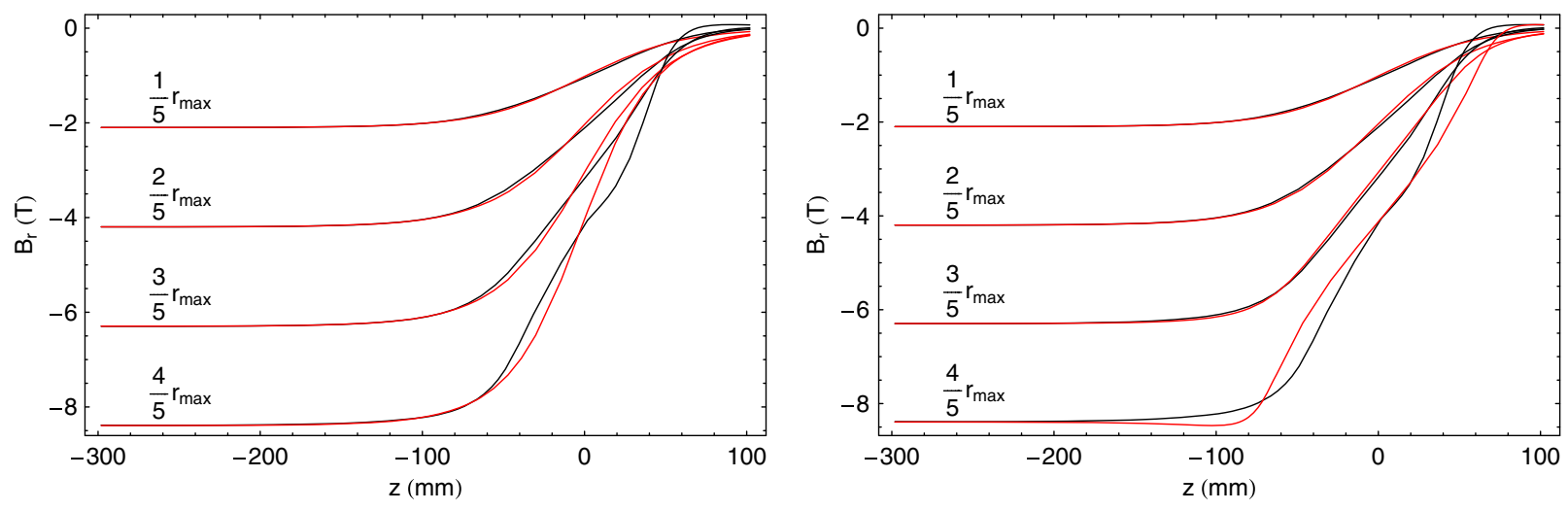

FIG. 10. As Fig. 7, but with $b_{2}=1.5$ (left) and $b_{2}=3.5$ (right).

field; but differences are apparent at larger distances from the axis. The parameter $b_{2}$ therefore allows control over the field behavior at large $r$, after the parameters $a_{0}, a_{1}$ and $a_{2}$ have been chosen to fit the field behavior close to the axis of the magnet.

Although the HL-LHC inner triplet quadrupoles are superconducting magnets, we can nevertheless inspect the shape of the pole face that would be needed to give the same field. Assuming poles with infinite magnetic permeability, the shape of a pole face corresponds to a surface of constant magnetic scalar potential $\varphi$. In the case of a quadrupole with gradient falling off as an Enge function, the scalar potential is given by (62): note that for the present case, this expression needs to be symmetrized so that the field has the symmetry $B_{x} \leftrightarrow B_{y}$ under $x \leftrightarrow y$. Figure 6 shows an equipotential surface for the analytical field model fitted to the numerical HL-LHC inner triplet quadrupole field data. The surface has been chosen so that the magnetic scalar potential has the (arbitrary) value $\varphi=0.25 \mathrm{~T} \mathrm{~m}$. We see that the equipotential surface has the shape that might be expected of an iron-dominated quadrupole, with the curved surface following a hyperbola in a plane of constant negative $z$, and the end of the pole being reasonably flat (close to a plane of constant $z \approx 0$ ).

\section{B. EMMA quadrupoles}

The quadrupole magnets in EMMA are iron-dominated, normal conducting magnets. The unusual feature of the EMMA quadrupoles is that the diameter of the aperture is comparable to the length of the magnet; this allows the accelerator to have a large transverse acceptance in a lattice consisting of magnets packed very close together. However, the relatively large aperture of the quadrupoles means that the gradient falls off rapidly from the center of the magnet. There is no appreciable distance along the axis for which the gradient is constant, and a realistic model of the field must include some representation of the fringe fields.

Given numerical field data from a magnetic model of an EMMA quadrupole, we can repeat the analysis used for the
HL-LHC inner triplet quadrupole in Sec. VII A. We again use an Enge function of the form (63) to represent the falloff of the gradient along the axis of the magnet. The field data cover an area closely approaching the pole tip (the field values are given on a rectangular grid, with transverse coordinates extending to $36 \mathrm{~mm}$ ). The results of fitting the parameters in the Enge function to field data along a line parallel to the axis (at different distances from the axis and fixed polar angle $\theta=\pi / 4$ ) are shown in Table II. We see that there is much larger variation in the parameters if the fit is performed at different distances from the axis, compared to the case of the HL-LHC inner triplet quadrupole. This suggests that the quality of the fit will not be as good. Making a direct comparison of the field based on the analytical formula with the numerical field data confirms that this is the case: see Figs. 11, 12 and 13. Inspecting Fig. 11 suggests the reason for the poor quality of the fit. The gradient initially falls off quite rapidly along the axis from the center of the magnet, but there is a long "tail" as the gradient approaches zero: this asymmetric behavior cannot be represented accurately using an Enge function with a small number of coefficients. Using a larger number of Enge coefficients improves the quality of the fit for the field at a given radius (and polar angle $\theta$ ), but then performing the integral of the quadrupole gradient $g(z)$ to find the function corresponding to $F(1, \zeta)$ in $(57)$ becomes difficult.

TABLE II. Parameters for a fit of an Enge function of the form (63), to the quadrupole gradient in an EMMA F quadrupole. The maximum radius $r_{\max }$ in the field data is $36 \mathrm{~mm}$.

\begin{tabular}{lccc}
\hline \hline Radius of fit & $a_{0}$ & $a_{1}$ & $a_{2}$ \\
\hline$r_{\max } / 10$ & 0.0138149 & -0.162670 & 11.2914 \\
$r_{\max } / 8$ & 0.0172756 & -0.163023 & 11.3031 \\
$r_{\max } / 6$ & 0.0230543 & -0.163794 & 11.3286 \\
$r_{\max } / 4$ & 0.0346675 & -0.166075 & 11.4036 \\
$r_{\max } / 3$ & 0.0463818 & -0.169480 & 11.5140 \\
$r_{\max } / 2$ & 0.0702287 & -0.180796 & 11.8694 \\
\hline \hline
\end{tabular}




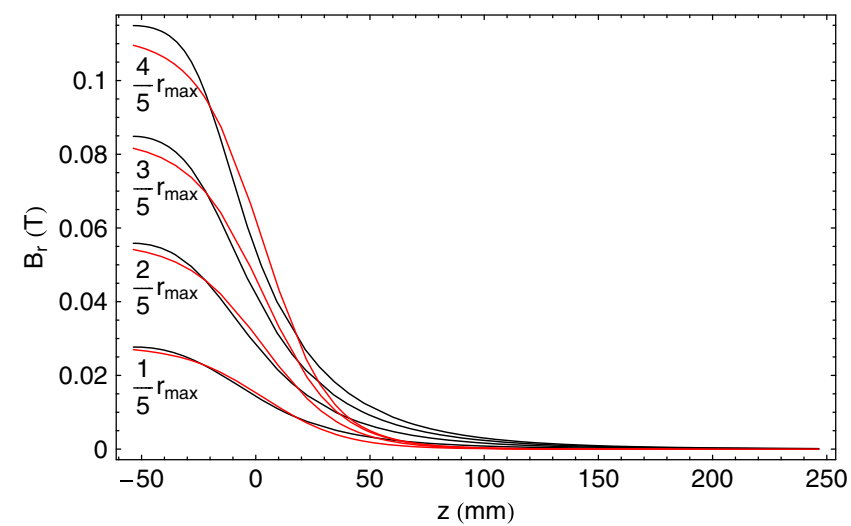

FIG. 11. Radial component of the magnetic field in an EMMA F quadrupole as a function of position along lines of given radius and with cylindrical polar coordinate $\theta=\pi / 4$. Each pair of black and red lines shows the field at a different radius, from $r_{\max } / 5$ to $4 r_{\max } / 5$, with $r_{\max }=36 \mathrm{~mm}$. The black lines show the field obtained from the (numerical) magnetic model; the red lines show a symmetrized form of the analytical model (49)-(51), with fit parameters given in Table II with radius of fit $r_{\max } / 10$, and $b_{2}=1.8$. The center of the quadrupole is at the far left of the plot, $z=-53.6 \mathrm{~mm}$.

It is of course possible to plot an equipotential surface to represent the shape of the pole in an EMMA quadrupole, in the same way that we did for a "normal conducting equivalent" HL-LHC inner triplet quadrupole. However, we find that the shape of the pole is much as expected for a normal conducting quadrupole, i.e., the plot is qualitatively very similar to that shown in Fig. 6.

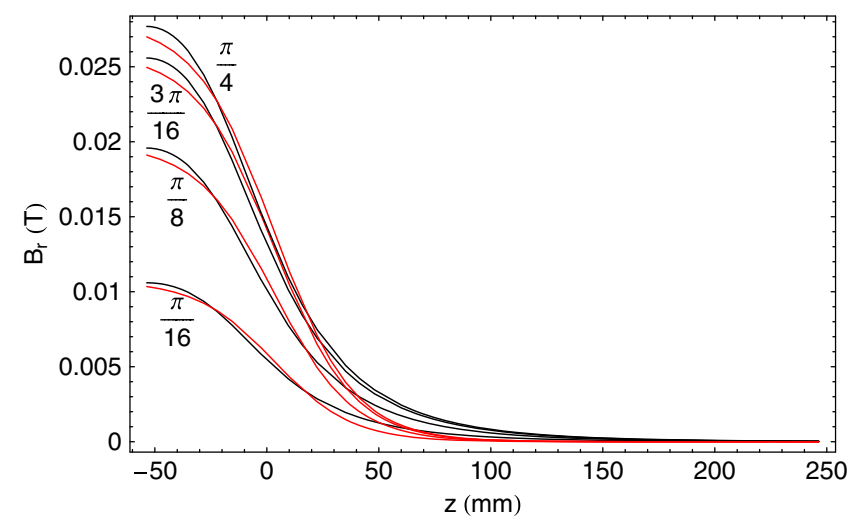

FIG. 12. Radial component of the magnetic field in an EMMA $\mathrm{F}$ quadrupole as a function of position along lines of fixed distance $r=r_{\max } / 4$. Each pair of black and red lines shows the field at a different value of the cylindrical polar coordinate $\theta$, from $\pi / 16$ to $\pi / 4$ (in steps of $\pi / 16$ ). The black lines show the field obtained from the (numerical) magnetic model; the red lines show a symmetrized form of the analytical model (49)-(51), with fit parameters given in Table II with radius of fit $r_{\max } / 10$, and $b_{2}=1.8$. The center of the quadrupole is at the far left of the plot, $z=-53.6 \mathrm{~mm}$.

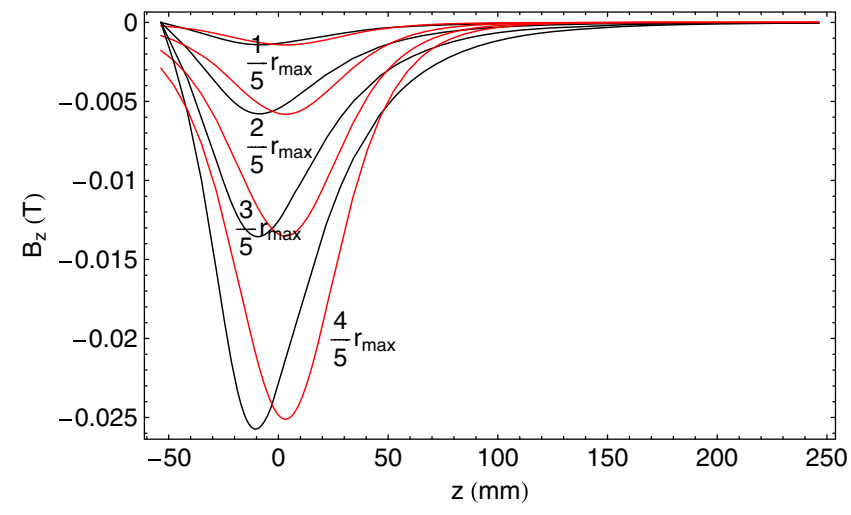

FIG. 13. Longitudinal component of the magnetic field in an EMMA F quadrupole as a function of position along lines of given radius and with cylindrical polar coordinate $\theta=\pi / 4$. Each pair of black and red lines shows the field at an increasing radius, from $r_{\max } / 5$ to $4 r_{\max } / 5$, with $r_{\max }=36 \mathrm{~mm}$. The black lines show the field obtained from the (numerical) magnetic model; the red lines show a symmetrized form of the analytical model (49)-(51), with fit parameters given in Table II with radius of fit $r_{\max } / 10$, and $b_{2}=1.8$. The center of the quadrupole is at the far left of the plot, $z=-53.6 \mathrm{~mm}$.

\section{CONCLUSIONS}

Closed-form analytic expressions have been presented for fringe fields in multipole magnets. For quadrupoles, the field described by the analytic expressions was shown to have the expected properties. The expressions can be extended to describe multipoles of any order. For ease of explanation and illustration, we looked in particular at fringe fields in which the multipole gradient had a falloff along the axis of the magnet described by an Enge function with only a single parameter in the exponent. However, the technique can be applied to any function with the appropriate dependence on the coordinates (i.e., any function that depends on the coordinates combined in the form $\sqrt{2} z \pm i h$ ). Examples of other (non-Enge) functions that may be suitable for describing fringe fields may be found in $[12,13]$.

Expressions were also given for scalar and vector potentials from which the multipole fringe fields presented here could be derived. Again, the expressions for the potentials can be extended to apply to multipole magnets of any order. The scalar potential is of interest since, in iron-dominated magnets, the pole faces form surfaces of constant scalar potential. This provides a connection between studies of the dynamics of particles moving through the fringe fields a particular magnet, and design studies of the magnet geometry. It is hoped that by having access to realistic analytical descriptions of fringe fields at an early stage in the design of an accelerator beam line, the design process (typically involving many iterations between beam dynamics studies and magnet design work) may be made more efficient.

The vector potential is of interest for particle tracking. In particular, some techniques for symplectic integration of the equations of motion for particles moving in magnetic 
fields are based on analytical expressions for the vector potential (see, for example, [20]). Again, it is hoped that there will be benefits in being able to perform symplectic tracking through realistic fringe field models at an early stage in the design of an accelerator.

In some types of magnets, such as those used in nonscaling FFAGs, fringe fields dominate the effects of the magnet. In such cases, being able to study the impact of fringe fields at an early stage of the accelerator design is essential for making efficient progress with the design. It should be possible to implement the methods presented here in standard accelerator tracking codes; this will allow accurate modeling of fringe field effects in multipole magnets of arbitrary order, and enhance the range of tools available for accelerator design and simulation.

\section{ACKNOWLEDGMENTS}

This work has been ongoing for many years and B. D. M. thanks Deepa Angal-Kalinin for giving him the opportunity and required support to finish it. B. D. M. is also pleased to acknowledge useful discussions on both form and content, as well as general encouragement over the years from the following: Jim Clarke, Chris Edmonds, Bas van der Geer, Fay Hannon, Werner Herr, David Holder, Marieke de Loos, Neil Marks, Giovanni Muratori Sr., Hywel Owen and Peter Williams. The authors would like to thank Susana Izquierdo Bermudez for the field data for the HL-LHC Inner Triplet quadrupoles, and Chris Edmonds for the field data for the EMMA quadrupole. This work was partially supported by the FP7 HiLumi LHC Design Study (http:// hilumilhc.web.cern.ch).

\section{APPENDIX: SOLUTION OF MAXWELL'S EQUATIONS}

In this Appendix, we show that a solution to the magnetostatic Maxwell's equations with the properties expected for a multipole fringe field takes the form (31)-(33). Based on a generalization of the fringe fields for the dipole case, we start from the assumption that multipole fringe fields will be of the form

$$
\begin{aligned}
& B_{u}=\frac{f_{1}(u, v, \zeta)+f_{2}(u, v, \zeta) e^{\zeta}}{1+2 f_{3}(u, v) e^{\zeta}+e^{2 \zeta}}, \\
& B_{v}=\frac{f_{4}(u, v, \zeta)+f_{5}(u, v, \zeta) e^{\zeta}}{1+2 f_{3}(u, v) e^{\zeta}+e^{2 \zeta}}, \\
& B_{\zeta}=\frac{f_{6}(u, v, \zeta)+f_{7}(u, v, \zeta) e^{\zeta}}{1+2 f_{3}(u, v) e^{\zeta}+e^{2 \zeta}},
\end{aligned}
$$

where the functions $f_{i}$ (for $i=1, \ldots, 7$ ) are arbitrary functions of the specified variables. As we shall see, this provides sufficient generality that the multipole gradient can decay along the $z$ axis in any desired fashion. Substituting (A1)-(A3) into Maxwell's equations (24)(27) gives a set of constraints on the possible forms of the functions $f_{1}, f_{2}$ etc. To obtain useful expressions for multipole fringe fields, we need to find solutions satisfying the various constraints.

Essentially, there are only two types of derivative that we need to consider. These are

$$
\begin{aligned}
\partial_{u} B_{u} & =\frac{\partial_{u} f_{1}+\partial_{u} f_{2} e^{\zeta}}{A}-\frac{2\left(f_{1}+f_{2} e^{\zeta}\right) e^{\zeta} \partial_{u} f_{3}}{A^{2}}, \\
\partial_{\zeta} B_{u} & =\frac{\partial_{\zeta} f_{1}+\partial_{\zeta} f_{2} e^{\zeta}+f_{2} e^{\zeta}}{A}-\frac{2\left(f_{1}+f_{2} e^{\zeta}\right)\left(e^{\zeta} f_{3}+e^{2 \zeta}\right)}{A^{2}},
\end{aligned}
$$

where $A=1+2 f_{3} e^{\zeta}+e^{2 \zeta}$. For the remaining derivatives, we simply implement the following changes sequentially:

$$
\begin{array}{lll}
\partial_{v} B_{u} \equiv \partial_{u} B_{u} & \text { under } & (u \leftrightarrow v), \\
\partial_{u} B_{v} \equiv \partial_{u} B_{u} & \text { under } & \left(f_{1} \rightarrow f_{4}, f_{2} \rightarrow f_{5}\right), \\
\partial_{v} B_{v} \equiv \partial_{u} B_{v} & \text { under } & (u \leftrightarrow v), \\
\partial_{\zeta} B_{v} \equiv \partial_{\zeta} B_{u} & \text { under } & \left(f_{1} \rightarrow f_{4}, f_{2} \rightarrow f_{5}\right), \\
\partial_{u} B_{\zeta} \equiv \partial_{u} B_{u} & \text { under } & \left(f_{1} \rightarrow f_{6}, f_{2} \rightarrow f_{7}\right), \\
\partial_{v} B_{\zeta} \equiv \partial_{u} B_{\zeta} & \text { under } & (u \leftrightarrow v), \\
\partial_{\zeta} B_{\zeta} \equiv \partial_{\zeta} B_{u} & \text { under } & \left(f_{1} \rightarrow f_{6}, f_{2} \rightarrow f_{7}\right) .
\end{array}
$$

As all equations (24)-(27) are equal to zero, we can take out a factor of $A^{2}$ to give

$$
\begin{gathered}
\left(\partial_{x} f_{1}+\partial_{x} f_{2} e^{\zeta}\right)\left(1+2 f_{3} e^{\zeta}+e^{2 \zeta}\right)+\left(\partial_{y} f_{4}+\partial_{y} f_{5} e^{\zeta}\right)\left(1+2 f_{3} e^{\zeta}+e^{2 \zeta}\right) \\
+\left(\partial_{\zeta} f_{6}+\partial_{\zeta} f_{7} e^{\zeta}+f_{7} e^{\zeta}\right)\left(1+2 f_{3} e^{\zeta}+e^{2 \zeta}\right) \\
-2\left(f_{1}+f_{2} e^{\zeta}\right) e^{\zeta} \partial_{x} f_{3}-2\left(f_{4}+f_{5} e^{\zeta}\right) e^{\zeta} \partial_{y} f_{3}-2\left(f_{6}+f_{7} e^{\zeta}\right)\left(f_{3} e^{\zeta}+e^{2 \zeta}\right)=0 \\
\left(\partial_{x} f_{4}+\partial_{x} f_{5} e^{\zeta}\right)\left(1+2 f_{3} e^{\zeta}+e^{2 \zeta}\right)-\left(\partial_{y} f_{1}+\partial_{y} f_{2} e^{\zeta}\right)\left(1+2 f_{3} e^{\zeta}+e^{2 \zeta}\right) \\
-2\left(f_{4}+f_{5} e^{\zeta}\right) e^{\zeta} \partial_{x} f_{3}+2\left(f_{1}+f_{2} e^{\zeta}\right) e^{\zeta} \partial_{y} f_{3}=0, \\
\left(\partial_{x} f_{6}+\partial_{x} f_{7} e^{\zeta}\right)\left(1+2 f_{3} e^{\zeta}+e^{2 \zeta}\right)-\left(\partial_{\zeta} f_{1}+\partial_{\zeta} f_{2} e^{\zeta}+f_{2} e^{\zeta}\right)\left(1+2 f_{3} e^{\zeta}+e^{2 \zeta}\right) \\
-2\left(f_{6}+f_{7} e^{\zeta}\right) e^{\zeta} \partial_{x} f_{3}+2\left(f_{1}+f_{2} e^{\zeta}\right)\left(f_{3} e^{\zeta}+e^{2 \zeta}\right)=0, \\
\left(\partial_{y} f_{6}+\partial_{y} f_{7} e^{\zeta}\right)\left(1+2 f_{3} e^{\zeta}+e^{2 \zeta}\right)-\left(\partial_{\zeta} f_{4}+\partial_{\zeta} f_{5} e^{\zeta}+f_{5} e^{\zeta}\right)\left(1+2 f_{3} e^{\zeta}+e^{2 \zeta}\right) \\
-2\left(f_{6}+f_{7} e^{\zeta}\right) e^{\zeta} \partial_{x} f_{3}+2\left(f_{4}+f_{5} e^{\zeta}\right)\left(f_{3} e^{\zeta}+e^{2 \zeta}\right)=0 .
\end{gathered}
$$


We can now equate coefficients of $e^{\zeta}$ giving

$$
\begin{array}{ll}
e^{3 \zeta}: \quad & \partial_{u} f_{2}+\partial_{\zeta} f_{7}-f_{7}=0 \\
& \partial_{v} f_{5}+\partial_{\zeta} f_{7}-f_{7}=0 \\
& \partial_{u} f_{7}-\partial_{\zeta} f_{5}+f_{5}=0 \\
& \partial_{v} f_{7}-\partial_{\zeta} f_{2}+f_{2}=0 \\
e^{2 \zeta}: \quad & f_{2} \partial_{u} f_{3}+f_{6}-f_{3} f_{7}=0 \\
& f_{5} \partial_{v} f_{3}+f_{6}-f_{3} f_{7}=0 \\
& f_{7} \partial_{u} f_{3}-f_{4}+f_{3} f_{5}=0 \\
& f_{7} \partial_{v} f_{3}-f_{1}+f_{3} f_{2}=0 \\
& \\
e^{\zeta}: \quad & f_{1} \partial_{u} f_{3}+f_{3} f_{6}-f_{7}=0 \\
& f_{4} \partial_{v} f_{3}+f_{3} f_{6}-f_{7}=0 \\
& f_{6} \partial_{u} f_{3}+f_{5}-f_{3} f_{4}=0 \\
& f_{6} \partial_{v} f_{3}+f_{2}-f_{3} f_{1}=0 \\
& \\
e_{u} f_{1}+\partial_{\zeta} f_{6}=0 \\
& \partial_{v} f_{4}+\partial_{\zeta} f_{6}=0 \\
& \partial_{u} f_{6}-\partial_{\zeta} f_{4}=0 \\
& \partial_{v} f_{6}-\partial_{\zeta} f_{1}=0 .
\end{array}
$$

Note that we have not included all the steps and the above equations represent the original set with all possible simplifications taking into account the set itself. Note also that Eqs. (A4)-(A7) and (A16)-(A19) may be solved independently of the rest and can therefore be dealt with later. From Eq. (A11), using (A8) and (A12), we see that

$$
f_{7}\left(\partial_{v} f_{3} \partial_{u} f_{3}+f_{3}^{2}-1\right)=0 .
$$

Had we looked at Eqs. (A10) and (A14) instead, using (A9) and (A13), we would have had

$$
f_{6}\left(\partial_{v} f_{3} \partial_{u} f_{3}+f_{3}^{2}-1\right)=0,
$$

with the same result from Eq. (A15). Now, $f_{6}$ and $f_{7}$ cannot both be zero as this would mean $B_{\zeta}=0$. Therefore, we must have

$$
\partial_{v} f_{3} \partial_{u} f_{3}+f_{3}^{2}-1=0 .
$$

The general solution for $f_{3}$, using the method of characteristics [14], is

$$
f_{3}=\sin h(u, v),
$$

with

$$
h(u, v)=\frac{1}{b} u+b v+c,
$$

where $b$ and $c$ are constant. Without loss of generality and for simplicity, we set $c=0$. Substituting this back into (A8)-(A15) gives the relations

$$
\begin{aligned}
& f_{2}=b^{2} f_{5}, \\
& f_{1}=b^{2} f_{4} .
\end{aligned}
$$

The equations reduce to just two independent equations that may be written as

$$
\begin{aligned}
& \frac{1}{b} f_{2} \cos h+f_{6}-f_{7} \sin h=0, \\
& f_{6} \cos h+\frac{1}{b} f_{2}-\frac{1}{b} f_{1} \sin h=0 .
\end{aligned}
$$

Using $f_{1}=b^{2} f_{4}$ and Eqs. (A16) and (A17) we see that we require

$$
b^{2} \partial_{u} f_{4}=\partial_{v} f_{4},
$$

which can again be solved by the method of characteristics to give $f_{4}=f_{4}(h, z)$. Using this with Eqs. (A18) and (A19) we see that $f_{6}=f_{6}(h, z)$. Similarly, $f_{2}=b^{2} f_{5}$ applied to (A4) and (A5) and, subsequently (A6) and (A7) gives $f_{5}=f_{5}(h, z)$ and $f_{7}=f_{7}(h, z)$. This leaves six equations to be satisfied from the original system (A4)-(A19), namely (A21) and (A22) together with

$$
\begin{gathered}
\partial_{u} f_{2}+\partial_{\zeta} f_{7}-f_{7}=0 \\
\partial_{v} f_{7}-\partial_{\zeta} f_{2}+f_{2}=0 \\
\partial_{u} f_{1}+\partial_{\zeta} f_{6}=0 \\
\partial_{v} f_{6}-\partial_{\zeta} f_{1}=0 .
\end{gathered}
$$

After cross differentiation, Eqs. (A25) and (A26) give

$$
\begin{aligned}
& \partial_{u, v}^{2} f_{6}+\partial_{\zeta}^{2} f_{6}=0, \\
& \partial_{u, v}^{2} f_{1}+\partial_{\zeta}^{2} f_{1}=0 .
\end{aligned}
$$

Now, we can reexpress the partial derivatives in $u$ and $v$ in terms of $h$ only. The equations simplify to

$$
\triangle f_{1}=\triangle f_{6}=0,
$$

where $\triangle \equiv \partial_{h}^{2}+\partial_{\zeta}^{2}$. We introduce the coordinates 


$$
\begin{aligned}
& w=h+i \zeta, \\
& \bar{w}=h-i \zeta .
\end{aligned}
$$

Note that this operation is equivalent to complex conjugation in the $\zeta$ coordinate only and the function $h$ is untouched. Therefore, we have the solutions

$$
\begin{aligned}
& f_{1}=p_{1}(w)+q_{1}(\bar{w}), \\
& f_{6}=p_{6}(w)+q_{6}(\bar{w}),
\end{aligned}
$$

where $p_{1}, p_{6}$ are arbitrary functions of $w$, and $q_{1}, q_{6}$ are arbitrary functions of $\bar{w}$. Substituting this back into (A25) and (A26), we see that the solutions are further constrained to

$$
f_{1}=-i b p_{6}+i b q_{6}+k
$$

with $k$ constant, from which we can get $f_{4}$ via $f_{4}=\frac{1}{b^{2}} f_{1}$. Subsequently, we can get $f_{2}$ from (A22) and hence $f_{5}$ via $f_{5}=\frac{1}{b^{2}} f_{2}$ and $f_{7}$ from (A21). The general result, in terms of $p_{6}$ and $q_{6}$ may be summarized as follows (with $\left.h=\frac{1}{b} u+b v\right)$ :

$$
\begin{gathered}
f_{1}=-i b p_{6}+i b q_{6}+k \\
f_{2}=\left(-i b p_{6}+i b q_{6}+k\right) \sin h-\left(b p_{6}+b q_{6}\right) \cos h \\
f_{3}=\sin h \\
f_{4}=\frac{1}{b}\left(-i p_{6}+i q_{6}+\frac{k}{b}\right),
\end{gathered}
$$$$
f_{5}=\frac{1}{b}\left(-i p_{6}+i q_{6}+\frac{k}{b}\right) \sin h-\frac{1}{b}\left(p_{6}+q_{6}\right) \cos h,
$$

$f_{7}=\left(p_{6}+q_{6}\right) \sin h+\left(-i p_{6}+i q_{6}+\frac{k}{b}\right) \cos h$.

We are left with Eqs. (A23) and (A24) to be solved. Upon substitution of (A27)-(A33), these are actually seen to be trivially satisfied with no further constraints on any of the functions $f_{i}$. In fact, the results constitute a Darboux transformation, where, given a solution to Maxwell's equations expressed by (A16)-(A19), a new solution may be created, given by (A1)-(A3), provided (A4)-(A15) are satisfied. Further, the results can be seen to imply the following solution to Maxwell's equations:

$$
\begin{aligned}
B_{u} & =-i b f(h+i \zeta)+i b g(h-i \zeta), \\
B_{v} & =-\frac{i}{b} f(h+i \zeta)+\frac{i}{b} g(h-i \zeta), \\
B_{\zeta} & =f(h+i \zeta)+g(h-i \zeta),
\end{aligned}
$$

with $h$ defined by (A20). Writing the arbitrary functions $f$ and $g$ as functions of $\zeta \pm i h$ rather than as functions of $h \pm i \zeta$ leads to the form of solution given in (31)-(33). This solution could have been arrived at by a shorter method, but the above workings show that, for the kind of functions we are interested in, it is the only solution that fits. We may have been forced to consider more complicated functions by introducing nonlinearities in the coordinate dependance, for example. Note that the constant $b$ is purely a scaling constant of the coordinates, as well as giving a proportionality between $B_{u}$ and $B_{v}$.

[1] S. B. van der Geer and M. J. de Loos, GPT (General Particle Tracer), http://www.pulsar.nl/.

[2] É. Forest, Beam Dynamics: A New Attitude and Framework (Harwood, Sidney, 1998).

[3] É. Forest and J. Milutinović, Leading order hard edge fringe field effects exact in $(1+\delta)$ and consistent with Maxwell's equations for rectilinear magnets, Nucl. Instrum. Methods Phys. Res., Sect. A 269, 474 (1988).

[4] S. Caspi, M. Helm, and L. J. Laslett, 3D field harmonics, Lawrence Berkeley Laboratory Internal Report No. LBL-30313, 1991.

[5] S. Caspi, M. Helm, L. J. Laslett, and V. O. Brady, An approach to 3D magnetic field calculation using numerical and differential algebra methods, Lawrence Berkeley Laboratory Internal Report No. LBL-32624, 1992.

[6] M. Bassetti and C. Biscari, Analytical formulae for magnetic multipoles, Part. Accel. 52, 221 (1996).

[7] Y. Cai and Y. Nosochkov, Dynamical effects due to fringe field of the magnet in circular accelerators, in Proceedings of the 21st Particle Accelerator Conference, Knoxville, TN, 2005 (IEEE, Piscataway, NJ, 2005).

[8] B. D. Muratori, S. B. van der Geer, and M. J. de Loos, Multipole fringe fields, in Proceedings of the 2nd International Particle Accelerator Conference, San Sebastián, Spain (EPS-AG, Spain, 2011).

[9] H. A. Enge, Focusing of Charged Particles, edited by A. Septier (Academic Press, New York, 1967), Vol. 2, pp. 203-264.

[10] S. B. van der Geer and M. J. de Loos, GPT (General Particle Tracer) manual and documentation for dipole and rectangular magnets in GPT, 2004.

[11] M. Berz, B. Erdélyi, and K. Makino, Fringe field effects in small rings of large acceptance, Phys. Rev. ST Accel. Beams 3, 124001 (2000), and references therein.

[12] S. Kato, An improved description of magnetic fringing field, Nucl. Instrum. Methods Phys. Res., Sect. A 540, 1 (2005).

[13] S. Kato, Three-dimensional magnetic field distribution function beyond the bore radius of quadrupole magnets, 
Nucl. Instrum. Methods Phys. Res., Sect. A 611, 1 (2009).

[14] H. T. H. Piaggio, An elementary treatise on differential equations and their applications (G. Bell and Sons, Ltd., London, 1948).

[15] E. T. Whittaker, A Course of Modern Analysis (Cambridge University Press, Cambridge, England, 1902).

[16] E. T. Whittaker, On the partial differential equations of mathematical physics, Math. Ann. 57, 333 (1903).

[17] P. M. Morse and H. Feshbach, Methods of Theoretical Physics (McGraw-Hill, New York, 1953), p. 1253.

[18] K. L. Brown and J. E. Spencer, Non-linear optics for the final focus of the single-pass collider, in Proceedings of PAC'81 (IEEE, Piscataway, 1981), pp. 2568-2570.
[19] A. Jonquière, Note sur la série $\sum_{n=1}^{\infty} \frac{x^{n}}{n^{s}}$, Bull. S.M.F. 17, 142 (1889).

[20] Y. K. Wu, É. Forest, and D. S. Robin, Explicit symplectic integrator for s-dependent static magnetic field, Phys. Rev. E 68, 046502 (2003).

[21] N. Marks, B. J. A. Shepherd, B. Leigh, F. Goldie, and M. J. Crawley, Development and adjustment of the EMMA quadrupoles, in Proceedings of the 11th European Particle Accelerator Conference, Genoa, 2008 (EPS-AG, Genoa, Italy, 2008).

[22] Y. Giboudot, Study of beam dynamics in NS-FFAG EMMA with dynamical map, Ph.D. thesis, Brunel University, 2011. 